$$
\begin{aligned}
& \text { طر احى كنترل كننده بيش بين غير خطى زمان بيوسته با حل دستخاه } \\
& \text { معادلات ديفر انسيل - جبرى با شر ايط مرزى به روش اختلال هموتويى } \\
& \text { آذر السادات شعبانى '، عليرضا فاتحى '، فهيمه سلطانيان ، رضا جميل نياء }
\end{aligned}
$$

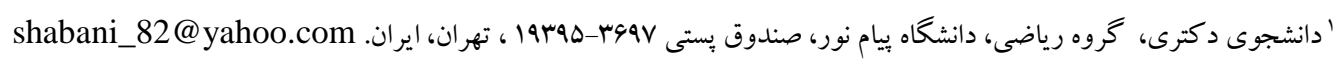

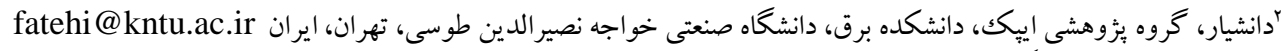

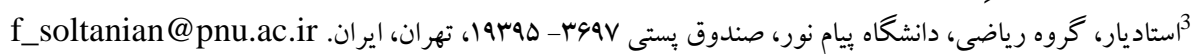

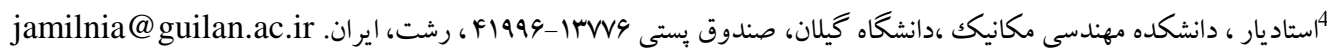

دريافت:

جكيده: در اين مقاله طراحى كنترل كننده بيشبين زمان ييو سته و حل معادلات ديفرانسيل-جبرى حاصل از آن با استفاده از روش نيمهتحليلى اختلال

هومو تويى ارائه شدهاست. در هر لحظه بروزرسانى الكوريتم كنترل بيش بين زمان بيوسته، مىبايست يك مساله كنترل بهينه حلقهباز حلشود. بهمنظور حل مساله كنترل بيش بين به صورت زمان بيوسته، مساله كنترل بهينه مذكور بايستى به روش غيرمستقيم حلشود. به اين ترتيب كه با بكار گيرى حساب تغييرات و اصل

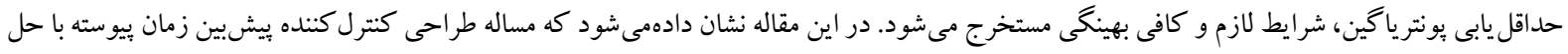
يكك دستگاه معادلات ديفر انسيل -جبرى با شرايط مرزى معادل است. براى حل اين دستگاه، روش نيمه تحليلى اختلال هموتويى ييشنهاد مى شود كه نتيجه آن بدست آمدن تابع كنترل و تابع حالت بهينه است. با داشتن اين توابع مى توان به آسانى از وضعيت حالت و كنترل در تمامى زمانها مطلع شد. روش ارائه شده مى تو اند براى طراحى كنترل كننده يِيشبين زمان بيوسته سيستمهاى خطى، غير خطى و همجنين سيستمهاى متغير بازمان بكار رود. به منظور نشان دادن قابليت و كارايى روش بيشنهادى جند مثال عددى همراه با شبيهسازى ارائه شدهاست. كلمات كليدى: كنترل بيشبين زمان-بيوسته، كنترل بهينه، اصل حداقل يابى بونترياگين، معادلات ديفر انسيل-جبرى، روش اختلال هموتويى

\title{
Design of nonlinear continuous time predictive controller by solving the differential-algebraic equations with boundary conditions using homotopy perturbation method
}

\begin{abstract}
Azar Shabani, Alireza Fatehi, Fahimeh Soltanian, Reza Jamilnia
Abstract: In this paper, design of continuous time predictive controller and solving the resulting differential-algebraic equations are presented using the semi-analytical homotopy perturbation method. At any updating time of the continuous time predictive control algorithm, an optimal open loop control problem must be solved. In order to solve the predictive control problem in continuous time, the problem of optimal control is solved by an indirect method. For this purpose, the necessary and sufficient conditions for optimality are determined by applying the variational calculus and the Pontryagin's minimum principle. A system of differential-algebraic equations with boundary conditions is created. Homotopy perturbation method is proposed to semi-analytically solve this problem. By specifying the control and the state functions, we can obtain easily the control and the state values in every instance of the prediction horizon. The presented method can be used to design of continuous-time predictive controller of linear, nonlinear and time varying systems. To illustrate the reliability and efficiency of the proposed method, some numerical examples with simulation results are presented.
\end{abstract}

Keywords: Continuous-Time Model Predictive Control, Optimal Control, Pontryagin's Minimum Principle, differential-algebraic equations, Homotopy Perturbation Method. 
اين منظور براى بهبود دقت گسستهازى و جلو گيرى از بكار گيرى تعداد

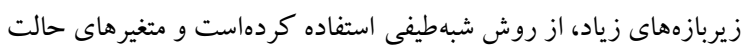

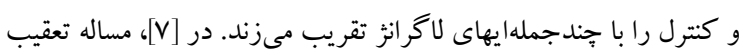

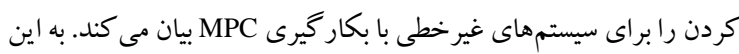

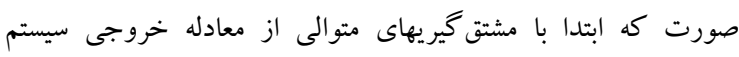
وبكار گيرى آنها در بسط تيلور خروجى سيستم، خطاى تعقيب كردن در

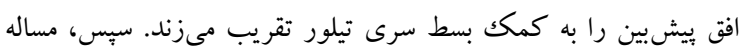

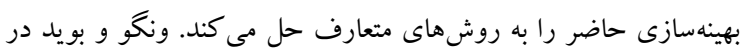

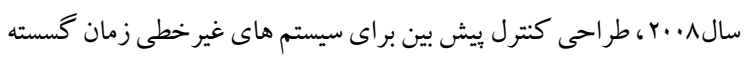

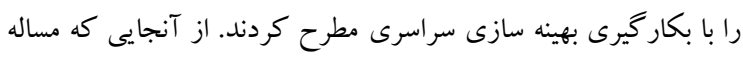

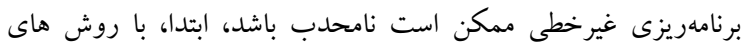

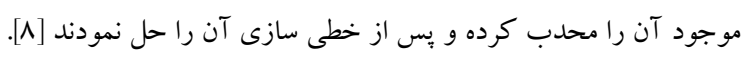

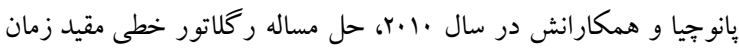

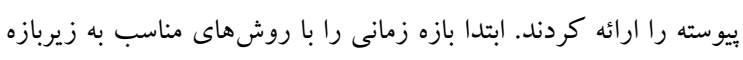

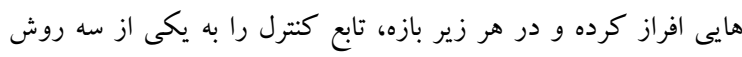

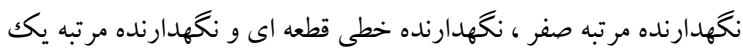
بارامترى نمودند. سبس، با تبديل مساله كنترل بهينه به فرم يكك مساله بهينه

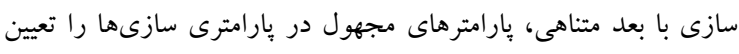

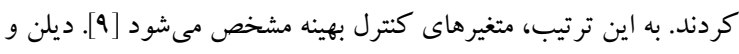

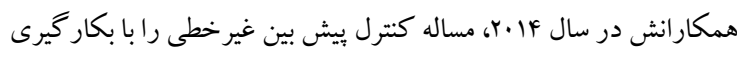

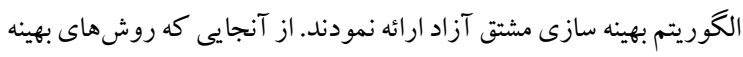

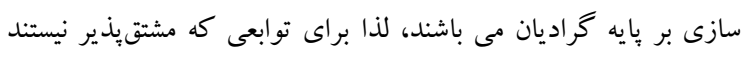

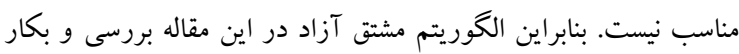

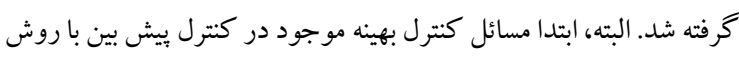

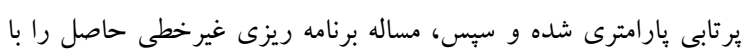

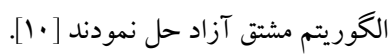

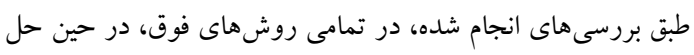

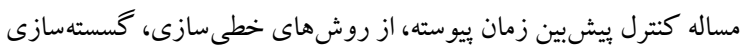

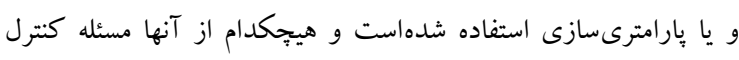
بيشبين را به صورت زمان بيوسته حل نكردهاست. از طرفى همانطور كه

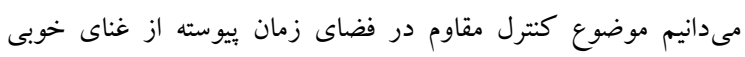

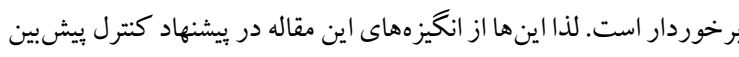

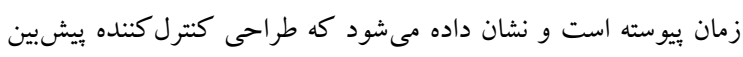
سيستمهاى غيرخطى زمان بيوسته، با يكك دستكاه معادلات ديفرانسيل

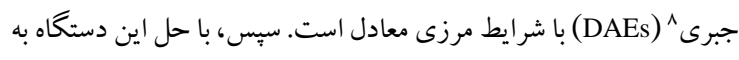

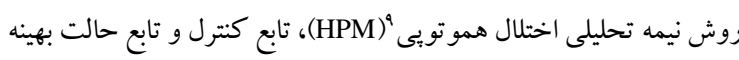

- (1) - مقدمه

بكك سيستم كنترل، ابزارى است كه از طريق توليد و اعمال

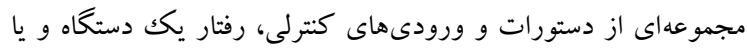

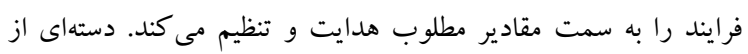
روشهاى كنترل نوين كه مدل سيستم حضور مشخص و فعالى در ساختار

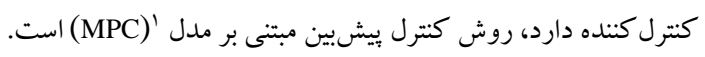
كنترل بيشبين، به يكك دسته از الكوريتمها اشاره دارد كه سيخنال

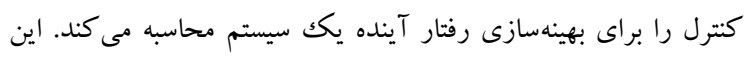

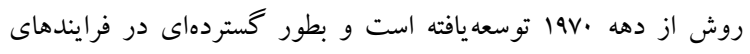

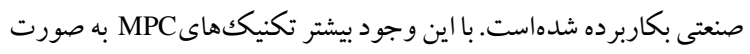

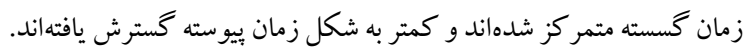
در سالهاى اخير، روشها و تكنيككهاى مختلفى براى حل مل مساله

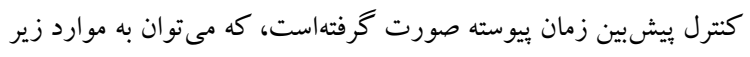

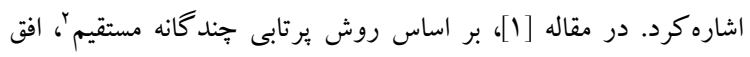

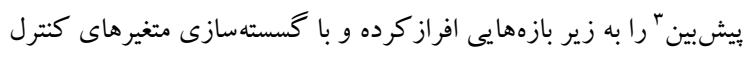

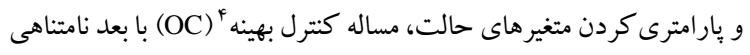

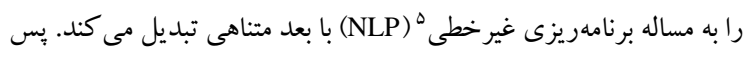

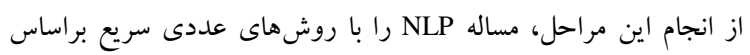

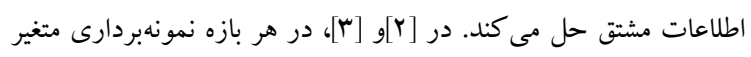

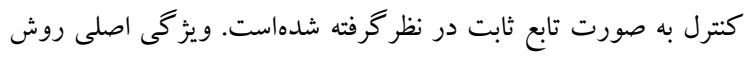

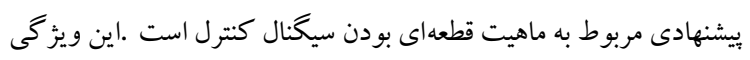

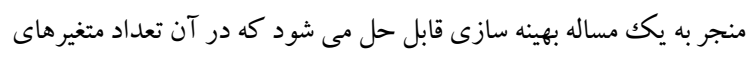

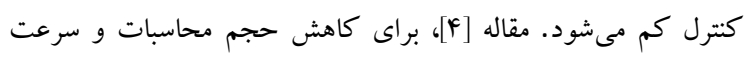

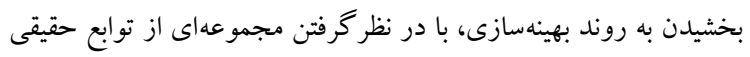

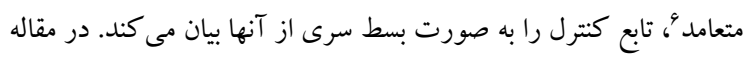

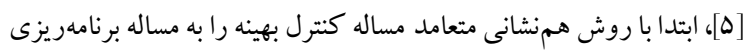
غير خطى تبديل مى كند. سبِ، براى يافتن مينيمم مطلق مساله بهينه سازى،

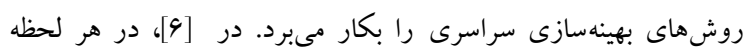
نمونهبردارى مساله كنترل ييشبين زمان ييو سته، مساله كنترل بهينه با استفاده

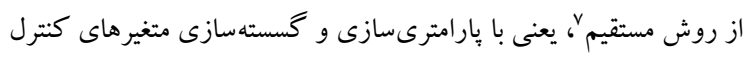

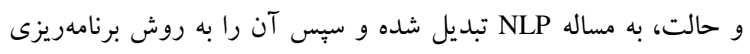
غيرخطى حل مى كند. در [9]، با اشاره به اين كه گسسته سازى مساله كنترل

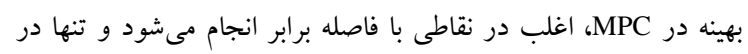

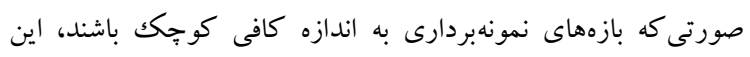

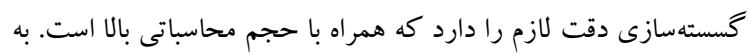

${ }^{v}$ Direct method

${ }^{\wedge}$ Differential-Algebraic Equations

${ }^{9}$ Semi analytical Homotopy Perturbation Method

\footnotetext{
${ }^{1}$ Model Predictive Control

${ }^{2}$ Multiple shooting direct method

${ }^{3}$ Predictive Horizon

${ }^{4}$ Optimal control

${ }^{5}$ Nonlinear programming problem

${ }^{6}$ Orthonormal functions
} 
فق لغزان مانند كنترل بيشبين استفاده نمود.

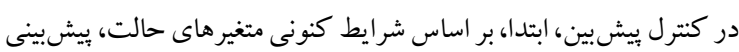

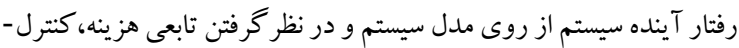

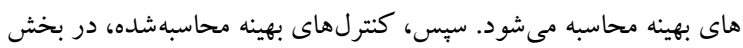

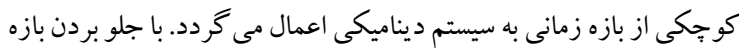

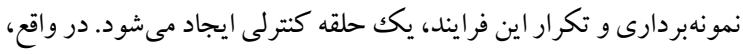
اين حلقه بسته كنترلى، از محاسبه كنترلهاى بهينه از شرايط لحظهاى

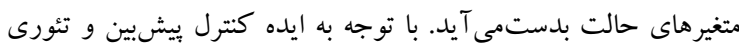

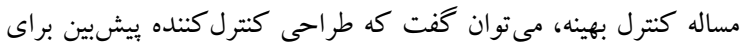

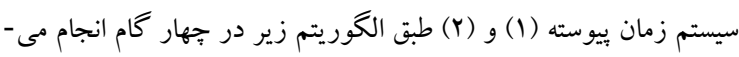

الكوريتم ا: ابتدا افق بيشبين HP، كام بروزرسانى 0 > ك ك،

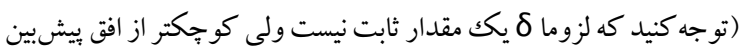
ti+1

$$
\text { ti t t t }
$$

-

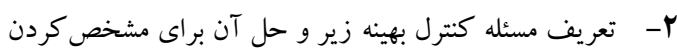

$$
\text { جواب }
$$

$\min _{\mathrm{u} \in \operatorname{pc}\left(\left[\mathrm{t}_{\mathrm{i}}, \mathrm{t}_{\mathrm{i}}+\mathrm{HP}\right], \mathrm{R}^{\mathrm{m}}\right)} \mathrm{J}_{\mathrm{HP}}(\mathrm{x}, \mathrm{u})$

$$
=\int_{t_{i}}^{t_{i}+H P} g(x(t), u(t), t) d t
$$

s.t $\quad \dot{x}(t)=f(x(t), u(t), t)$

$$
x\left(t_{i}\right)=x_{t_{i}}
$$

كه

$$
\text { , } \alpha:[a, b] \rightarrow \mathbb{R}^{m}
$$

t $\in$ بر سيستم در بازه u u

( $\left[t_{i}, t_{i}+\delta\right)$

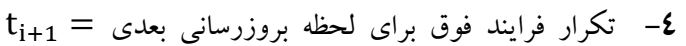

$$
t_{i}+\delta
$$

\section{r- مساله كنترل بهينه و رويكردهاى حل حل آن}

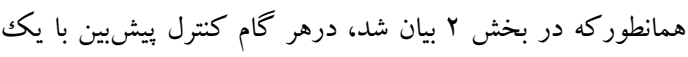

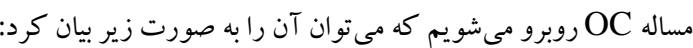
$\min J=\int_{t_{0}}^{t_{f}} g(x(t), u(t), t) d t$

$$
\begin{array}{cc}
\text { s.t } \quad \dot{x}(t)=f(x(t), u(t), t) \\
x\left(t_{0}\right)=x_{0}
\end{array}
$$

uد است كه باعث شود سيستم، تابع حالت قابل $\left[\mathrm{Cu}^{*}{ }_{1}(\mathrm{t}), \ldots, \mathrm{u}_{\mathrm{m}}{ }_{\mathrm{m}}(\mathrm{t})\right]$
به صورت زمان ييوسته مشخص مىشود. ساختار كلى بخشهاى مقاله به اين صورت است كه ابتدا مساله مورد

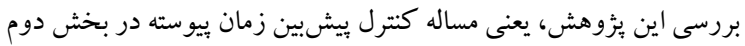

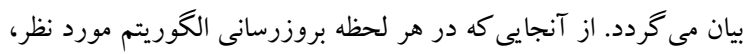

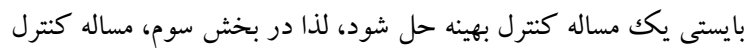
بهينه و رويكردهاى حل آن باز گومىشود. از طرفى براى حل مساله كنترل بهينه، روش غيرمستقيم' بكاربرده شدهاست، كه منجر به يك دوس دستخاه

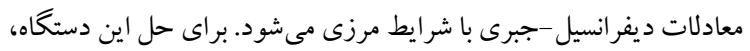
روش نيمه تحليلى اختلال هموتويى استفاده شدهاست كه در بخش جهر بهارم

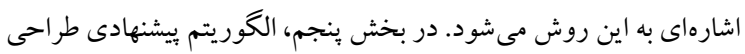

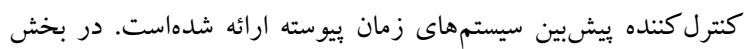

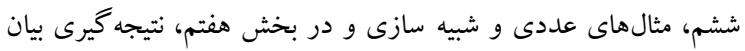

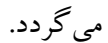

\section{r- بيان مساله كنترل بيشبين زمان بيوسته}

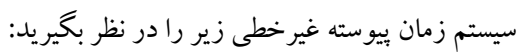

$$
\left\{\begin{array}{l}
\dot{x}(t)=f(x(t), u(t), t) \\
y(t)=k(x(t)) \\
x\left(t_{0}\right)=x_{0}
\end{array}\right.
$$

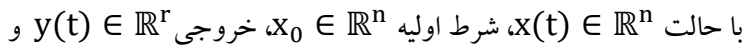
ورودى كنترل u(t)

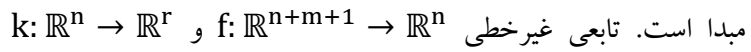

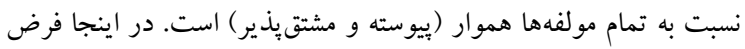
مىشود سيستم (1) براى هر شرط اوليه u: $\mathbb{R}^{+} \rightarrow U$

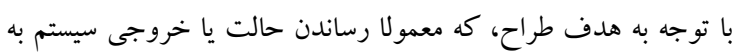

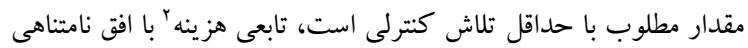

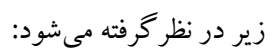

$$
J_{\infty}\left(x_{0}, u\right)=\int_{0}^{\infty} g(x(t), u(t), t) d t
$$

كه

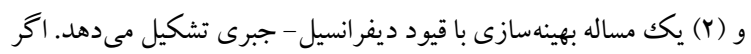
اين مساله بهينه سازى بتواند براى افق نامتناهى حل شود و همجنين هيج

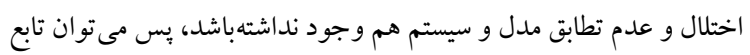

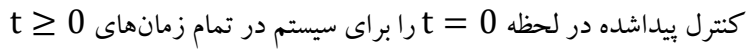
بكار برد. در حالت كلى جنين نيست و به خاطر اغتشاشات و عدم تطابق

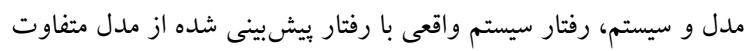

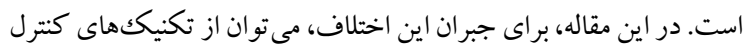




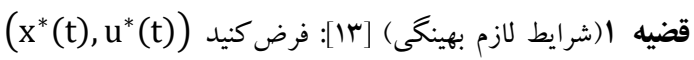

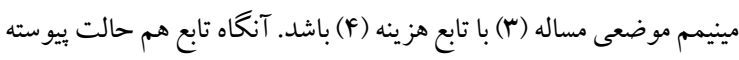

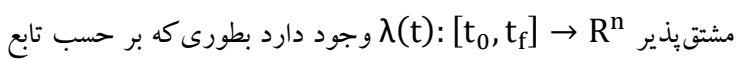
هميلتونين: - n $\mathcal{H}(\mathrm{x}(\mathrm{t}), \mathrm{u}(\mathrm{t}), \lambda(\mathrm{t}), \mathrm{t}) \triangleq$ $g(x(t), u(t), t)+\lambda^{T}(t)[f(x(t), u(t), t)]$

روابط زير برقرار است:

$$
\left\{\begin{array}{l}
\dot{\mathrm{x}}^{*}=\frac{\partial \mathcal{H}}{\partial \lambda}\left(\mathrm{x}^{*}(\mathrm{t}), \mathrm{u}^{*}(\mathrm{t}), \lambda^{*}(\mathrm{t}), \mathrm{t}\right) \\
\dot{\lambda}^{*}=-\frac{\partial \mathcal{H}}{\partial \mathrm{x}}\left(\mathrm{x}^{*}(\mathrm{t}), \mathrm{u}^{*}(\mathrm{t}), \lambda^{*}(\mathrm{t}), \mathrm{t}\right) \\
\mathcal{H}\left(\mathrm{x}^{*}(\mathrm{t}), \mathrm{u}^{*}(\mathrm{t}), \lambda^{*}(\mathrm{t}), \mathrm{t}\right) \leq \mathcal{H}\left(\mathrm{x}^{*}(\mathrm{t}), \mathrm{u}(\mathrm{t}), \lambda^{*}(\mathrm{t}), \mathrm{t}\right) \\
\quad \text { for all admissible } \mathrm{u}(\mathrm{t}) \in \mathrm{U}
\end{array}\right.
$$$$
\begin{aligned}
& {\left[\frac{\partial \mathrm{h}}{\partial \mathrm{x}}\left(\mathrm{x}^{*}\left(\mathrm{t}_{\mathrm{f}}\right), \mathrm{t}_{\mathrm{f}}\right)-\lambda^{*}\left(\mathrm{t}_{\mathrm{f}}\right)\right]^{\mathrm{T}} \delta \mathrm{x}_{\mathrm{f}}+} \\
& {\left[\mathcal{H}\left(\mathrm{x}^{*}\left(\mathrm{t}_{\mathrm{f}}\right), \mathrm{u}^{*}\left(\mathrm{t}_{\mathrm{f}}\right), \lambda^{*}\left(\mathrm{t}_{\mathrm{f}}\right) \mathrm{t}_{\mathrm{f}}\right)+\frac{\partial \mathrm{h}}{\partial \mathrm{t}}\left(\mathrm{x}^{*}\left(\mathrm{t}_{\mathrm{f}}\right), \mathrm{t}_{\mathrm{f}}\right)\right] \delta \mathrm{t}_{\mathrm{f}}=0}
\end{aligned}
$$

رابطه $\mathcal{H}\left(\mathrm{x}^{*}(\mathrm{t}), \mathrm{u}^{*}(\mathrm{t}), \lambda^{*}(\mathrm{t}), \mathrm{t}\right) \leq \mathcal{H}\left(\mathrm{x}^{*}(\mathrm{t}), \mathrm{u}(\mathrm{t}), \lambda^{*}(\mathrm{t}), \mathrm{t}\right)$ تاكيد بر "اصل حداقل يابى يونترياگين" دارد، كه (t) بايد تابع هميلتونين (ش) $\mathcal{H}\left(\mathrm{x}^{*}(\mathrm{t}), \mathrm{u}(\mathrm{t}), \lambda^{*}(\mathrm{t}), \mathrm{t}\right)$

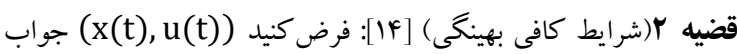

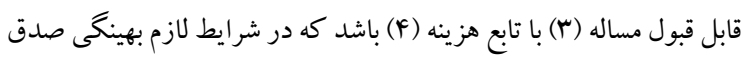
مى كند. اكر و مشتقيذير و محدب باشند آنكاه يك مينيمم مطلق مساله كترل بهينه است.

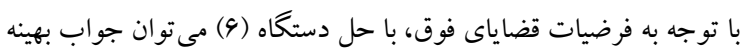

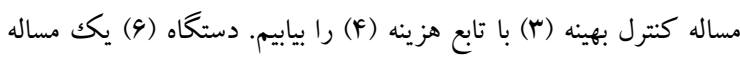

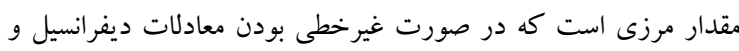

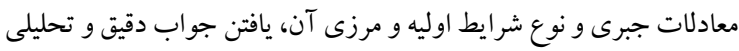

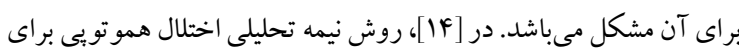

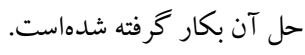

\section{ع-روش اختلال هموتويى}

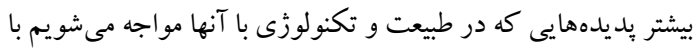

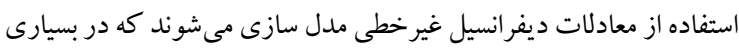

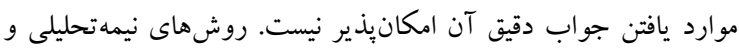

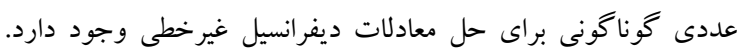

قبول

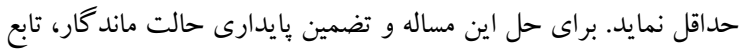

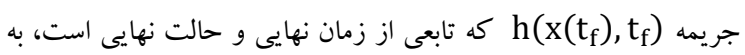

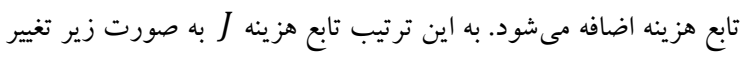

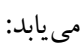

$$
J=h\left(x\left(t_{f}\right), t_{f}\right)+\int_{t_{0}}^{t_{f}} g(x(t), u(t), t) d t
$$

تعريف ا: زوج (x(·) (·) اكر در قيود مساله صدق نمايد.

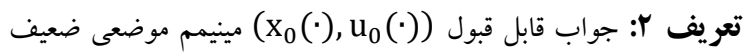

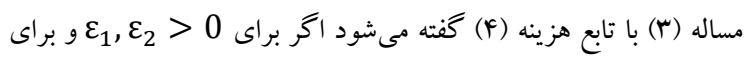

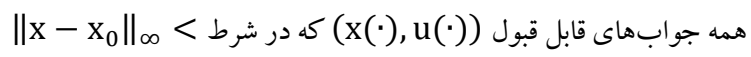
ع $\varepsilon_{1},\left\|u-u_{0}\right\|_{\infty}<\varepsilon_{2}$

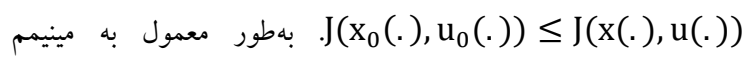

$$
\text { موضعى، جواب بهينه نيز تفته مىشود. }
$$
تعريف rا: جواب (x)

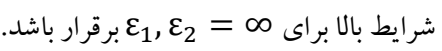

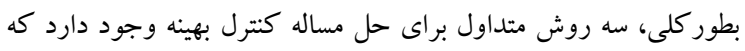

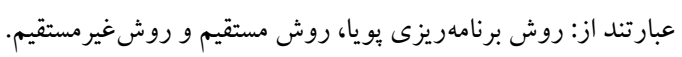

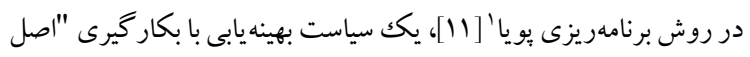

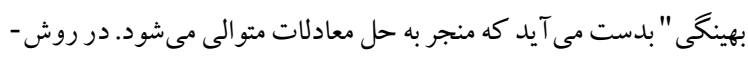

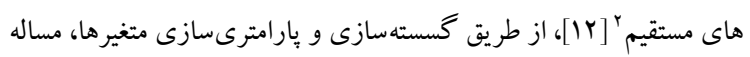

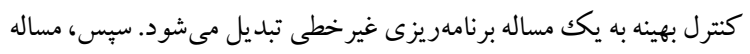

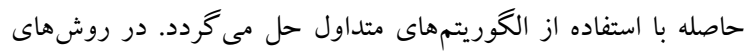

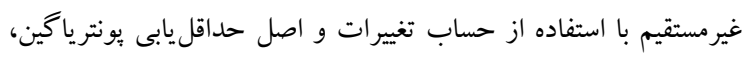

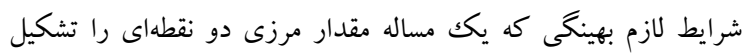

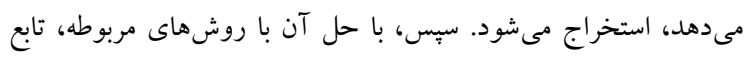

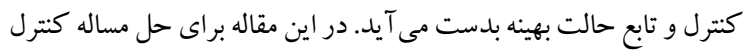

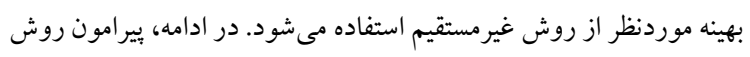
غيرمستقيم قضايايى بيان مىشود.

\section{1-" روش غير مستقيم حل مساله كنترل بهينه [ و • •}

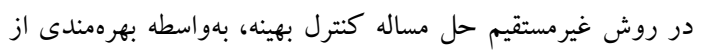

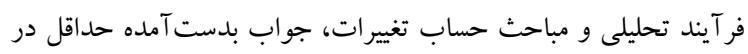

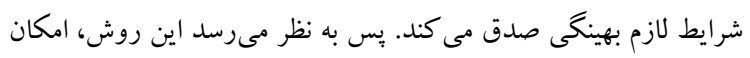
ارائه ياسخ بهينه با دقت بالا را در ميان روش هاى حلى حل مساله كتترل بهينه دارا

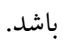


كه . توابع مجهول هستند كه طبق روش اختلال

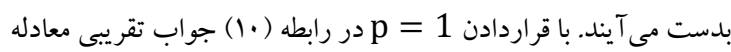

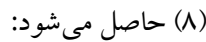

$$
x(t)=\lim _{p \rightarrow 1} v=v_{0}+v_{1}+v_{2}+\cdots
$$

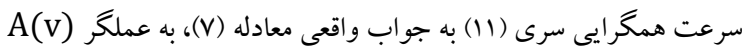

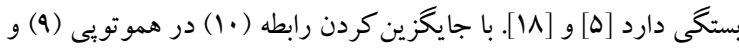

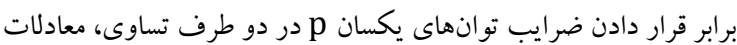

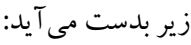

$\mathcal{L}\left(v_{m}(t)\right)$ $=-\aleph_{m-1}\left(v_{0}(t), v_{1}(t), \ldots, v_{m-1}(t)\right), m$

$\geq 1, \quad \mathcal{B}\left(v_{m}\right)=0$

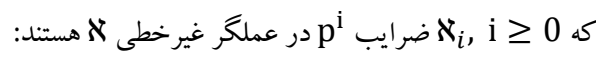

$\aleph(\mathrm{v}(\mathrm{t}))$

$=\aleph_{0}\left(\mathrm{v}_{0}(\mathrm{t})\right)+\mathrm{p} \aleph_{1}\left(\mathrm{v}_{0}(\mathrm{t}), \mathrm{v}_{1}(\mathrm{t})\right)$

$+\mathrm{p}^{2} \aleph_{2}\left(\mathrm{v}_{0}(\mathrm{t}), \mathrm{v}_{1}(\mathrm{t}), \mathrm{v}_{2}(\mathrm{t})\right)+\cdots$

كه (l با حل معادلات (Ir) به آسانى بدست مى آيند.

\section{0- روش يِينهادى براى حل مساله كنترل ييشبين غيرخطى زمان ييوسته}

همانطور كه در بخش (Y) بيان شد، طبق وليق الكوريتم 1، مساله كنترل

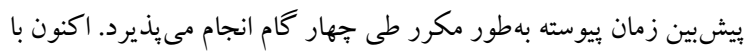

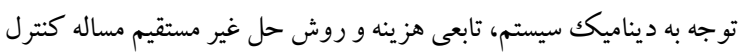

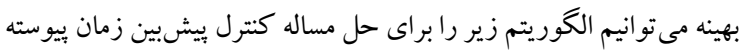

ارائه دهيم. تئن

تابعى هزينه و سيستم ديناميكى زير رادر نظر بخيريد [19]:

$$
J=h(x(T), T)+\int_{t_{0}}^{T} g(x(t), u(t), t) d t
$$$$
\dot{x}(t)=f(x(t), u(t), t)
$$

$y(t)=k(x(t))$

$x\left(t_{0}\right)=x_{0}$

طراحى كنترل كنتده بيشبين براى مساله (If)، كه يك كنترلكنتده

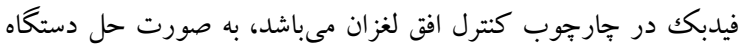

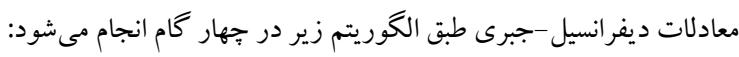
الكوريتم rا: ابتدا افق بيشبين HP، كام بروزرسانى 0 > (توجه كنيد كه لزوما $\delta$ يكك مقدار ثابت نيست ولى كوجکكتر از افق ييشبين تعريف مىشود) و دنباله لحظات بروزرسانى

$$
\text { t ti }
$$

روش نيمه تحليلى اختلال هموتويى، براى حل معادلات غيرخطى[10]

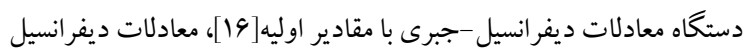
با مشتقات جزئى [IV] و ... بكار گرفته شدهاست.

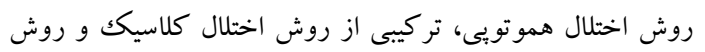

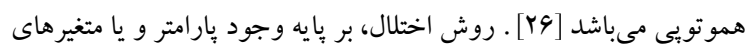

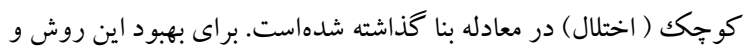

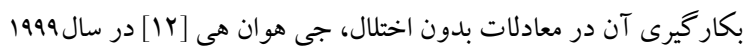

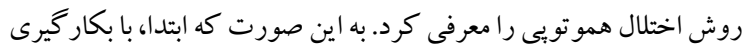

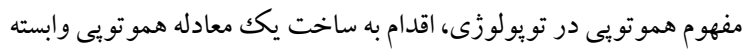
به معادله مورد نظر كرد. در اين معادله هموتويى، يار امتر اختلال وجود دود دارد

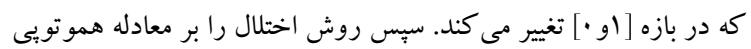

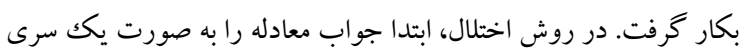

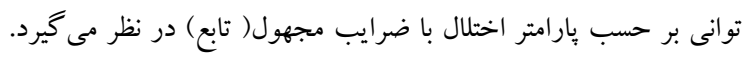

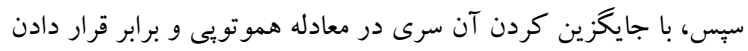

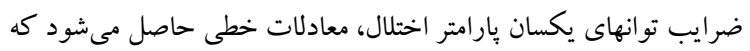
با حل دنبالهوار آنها توابع مجهول در سرى بدست مي بـآيند. براى توضيح

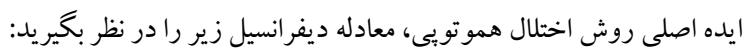

$$
A(x(t))-f(t)=0, \mathcal{B}(x)=0, t \in \Omega
$$

كه A عملكر ديفرانسيل كلى، B عملكر مرزى و f(t) يك تابع تحليلى

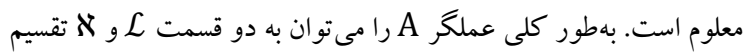
كرد، كه L عملكر خطى و א عملكر غير خطى است. بنابراين معادله (v) را مىتوان به صورت زير بازنويسى كرد:

$$
\mathcal{L}(x(t))+\aleph(x(t))-f(t)=0, \mathcal{B}(x)=0
$$

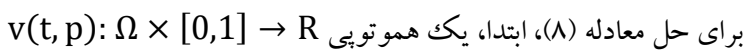
كه در شرط زير صدق مى كند ساخته مىشود: $\mathcal{H}(\mathrm{v}, \mathrm{p})=\mathcal{L}(\mathrm{v}(\mathrm{t}, \mathrm{p}))-\mathcal{L}\left(\mathrm{x}_{0}(\mathrm{t})\right)+\mathrm{p} \mathcal{L}\left(\mathrm{x}_{0}(\mathrm{t})\right)$ $+\mathrm{pw}(\mathrm{v}(\mathrm{t}, \mathrm{p}))-\mathrm{pf}(\mathrm{t})=0$

كه v(t,p) يك تابع مجهول، [0,1 p يك يارامتر جانشانى و

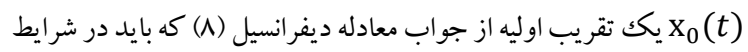

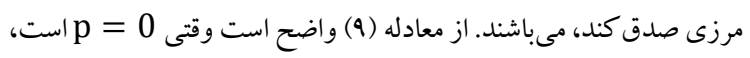
$v(t, 1)=x(t)$ مي $v(t, 0)=x_{0}(t)$

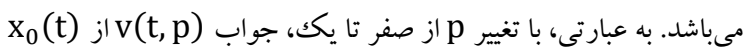

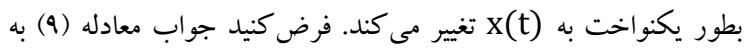

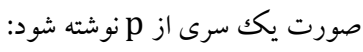
$v=v_{0}+p v_{1}+p^{2} v_{2}+\cdots$ 
نكته ا: براى يِياده سازى كتترل كننده طراحى شده زمان ويبوسته آن

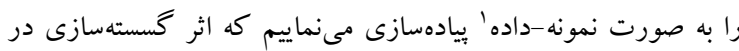

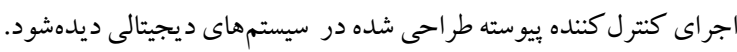

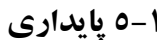

در MPC، يكك مساله كنترل بهينه افق نامتناهى با دنبالهاى از مسائل افق

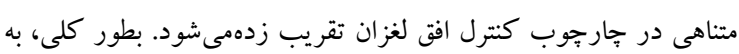

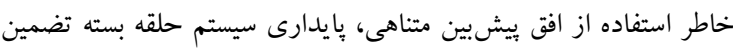

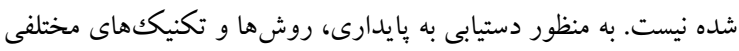

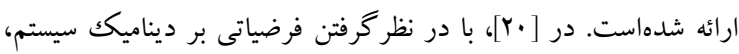

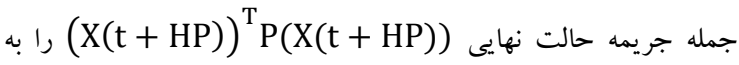

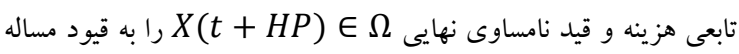

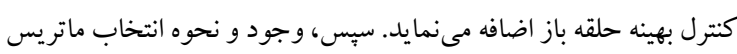
جريمه حالت نهايى P و ناحيه نهايى $\Omega$ به كونهاى كه شرط بايايدارى حلقه

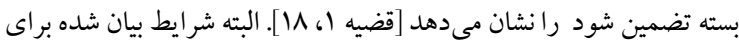

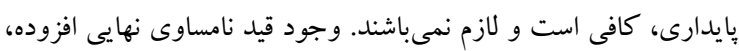

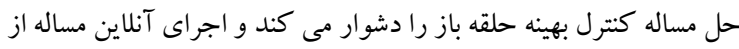

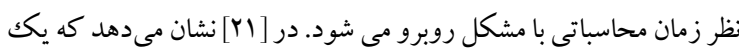

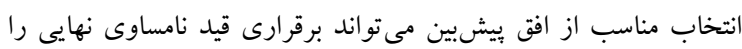

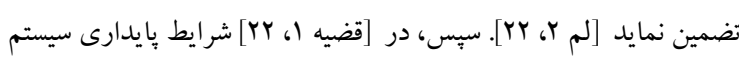

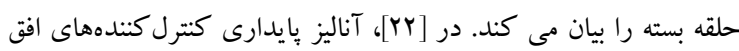

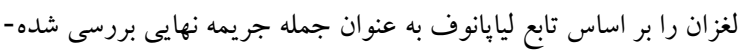

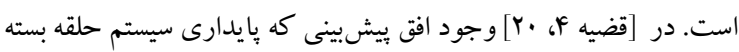

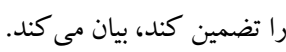

\section{7- مثال هاى عددى و شبيهسازى}

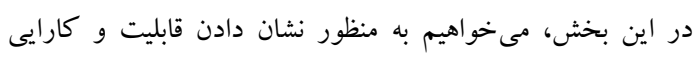

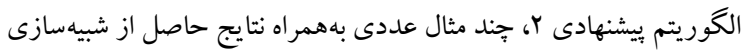

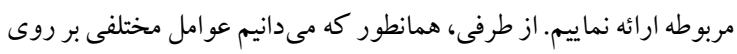

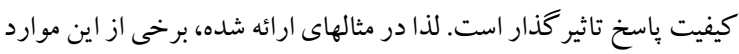

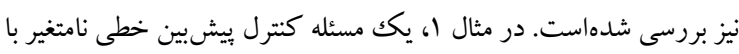

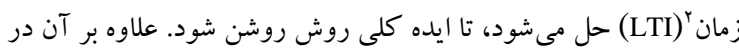

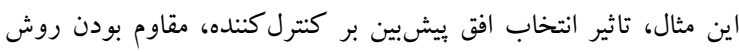

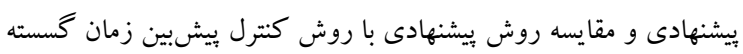

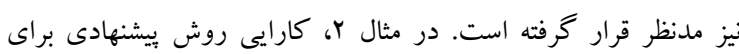

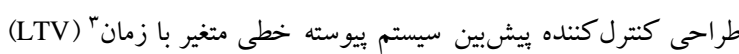

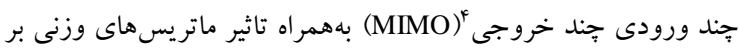

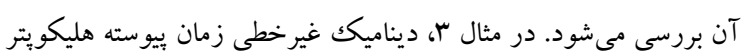

1- حل دستكاه معادلات ديفرانسيل-جبرى (DAEs) زير به روش

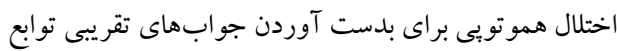
حالت $:\left[\mathrm{t}_{\mathrm{i}}, \mathrm{t}_{\mathrm{i}}+\mathrm{HP}\right)$ ( د) $\mathrm{u}_{\mathrm{MPC}}(\mathrm{t})$

$$
\left\{\begin{array}{l}
\dot{x}=f(x(t), u(t), t) \\
\dot{\lambda}=-\left[\frac{\partial f}{\partial x}(x(t), u(t), t)\right]^{T} \lambda(t)- \\
\quad \frac{\partial g}{\partial x}(x(t), u(t), t) \\
0=\frac{\partial g}{\partial u}(x(t), u(t), t)+\left[\frac{\partial f}{\partial u}(x(t), u(t), t)\right]^{T} \lambda(t) \\
x\left(t_{i}\right)=x_{i} \\
\lambda\left(t_{i}+H P\right)=\frac{\partial h}{\partial x}\left(x\left(t_{i}+H P\right)\right)
\end{array}\right.
$$

در روش اختلال هموتويى، ابتدا متناظر با هر يك از معادلات (جبرى (مان

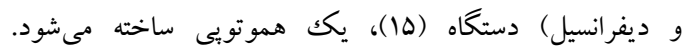

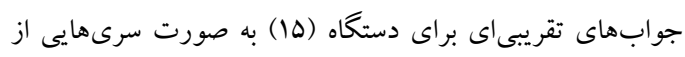

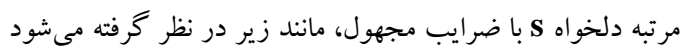

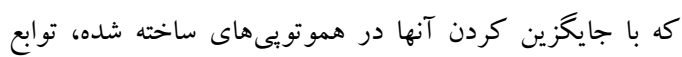
مجهول سرى بدست مى آيند:

$\left\{\begin{array}{l}\mathrm{x}(\mathrm{t}) \cong \hat{\mathrm{x}}(\mathrm{t})=\mathrm{x}_{0}(\mathrm{t})+\mathrm{px} \mathrm{x}_{1}(\mathrm{t})+\cdots+\mathrm{p}^{\mathrm{s}} \mathrm{x}_{\mathrm{s}}(\mathrm{t}) \\ \lambda(\mathrm{t}) \cong \hat{\lambda}(\mathrm{t})=\lambda_{0}(\mathrm{t})+\mathrm{p} \lambda_{1}(\mathrm{t})+\cdots+\mathrm{p}^{\mathrm{s}} \lambda_{\mathrm{s}}(\mathrm{t}) \\ \mathrm{u}(\mathrm{t}) \cong \hat{\mathrm{u}}(\mathrm{t})=\mathrm{u}_{0}(\mathrm{t})+\mathrm{pu} \mathrm{u}_{1}(\mathrm{t})+\cdots+\mathrm{p}^{\mathrm{s}} \mathrm{u}_{\mathrm{s}}(\mathrm{t})\end{array}\right.$

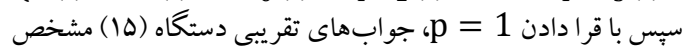

$\mathrm{x}_{\mathrm{MPC}}(\mathrm{t})=\mathrm{x}_{0}(\mathrm{t})+\mathrm{x}_{1}(\mathrm{t})+\cdots+\mathrm{x}_{\mathrm{s}}(\mathrm{t})$

$\lambda_{\text {MPC }}(\mathrm{t})=\lambda_{0}(\mathrm{t})+\lambda_{1}(\mathrm{t})+\cdots+\lambda_{\mathrm{s}}(\mathrm{t})$

$\mathrm{u}_{\text {MPC }}(\mathrm{t})=\mathrm{u}_{0}(\mathrm{t})+\mathrm{u}_{1}(\mathrm{t})+\cdots+\mathrm{u}_{\mathrm{s}}(\mathrm{t})$

$t \in\left[t_{i}, t_{i}+u_{\text {MPC }}(t)\right.$ بر سيستم در بازئ (ס و ناديده كرفتن باقيمانده سيكنال كنترل.

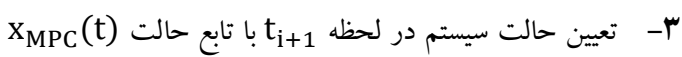

$$
\text { بلدست آمده در كام (1)، }
$$

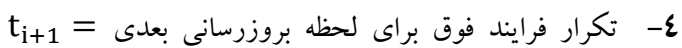

$$
t_{i}+\delta
$$

براى حل دستكاه (ها) به روش اختلال هموتويى، مقادير اوليه

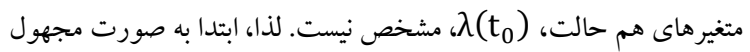
ل $\lambda\left(t_{0}\right)=\alpha, \alpha \in R^{n}$ $\lambda\left(t_{i}+H P\right)=$ دستكاه، مقادير مجهول $\alpha$ با توجه به شرط مرزى

$$
\text { بدست مى آيند. }
$$

$\begin{array}{ll}{ }^{3} \text { Linear time varying } & \text { ' Sampled Data } \\ { }^{4} \text { Multi Input Multi Output } & { }^{2} \text { Linear Time Invariant }\end{array}$ 
جواب نيمه تحليلى مرتبه S دستگاه (YI)، طبق روش اختلال هموتويى به صورت زير در نظر خرفته مىشود:

$\int \mathrm{x}(\mathrm{t}) \cong \hat{\mathrm{x}}(\mathrm{t})=\mathrm{x}_{0}(\mathrm{t})+\mathrm{px}_{1}(\mathrm{t})+\cdots+\mathrm{p}^{\mathrm{s}} \mathrm{x}_{\mathrm{s}}(\mathrm{t})$

$\lambda(t) \cong \hat{\lambda}(t)=\lambda_{0}(t)+p \lambda_{1}(t)+\cdots+p^{s} \lambda_{s}(t)$

( $r$ Y)

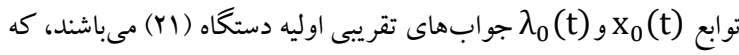

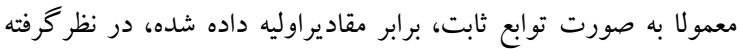
مىشوند يعنى بايد به گونهاى مشخصشوند كه در $\lambda_{i}(t), X_{i}(t), i=0, \ldots, s$

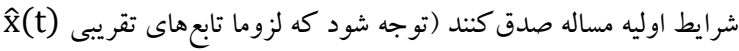

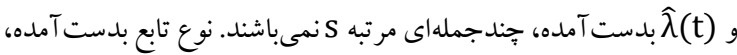

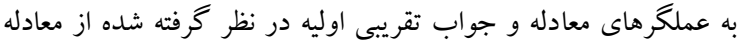

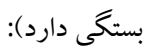

$$
\left\{\begin{aligned}
& \hat{x}\left(t_{0}\right)=x_{0}: \text { if } x_{0}(t)=x_{0} \text { then } \\
& x_{1}\left(t_{0}\right)=\cdots=x_{s}\left(t_{0}\right)=0 \\
& \hat{\lambda}\left(t_{0}\right)=\alpha: \quad \text { if } \lambda_{0}(t)=\alpha \text { then } \\
& \lambda_{1}\left(t_{0}\right)=\cdots=\lambda_{s}\left(t_{0}\right)=0
\end{aligned}\right.
$$

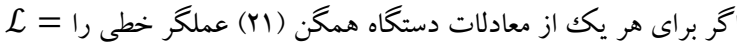

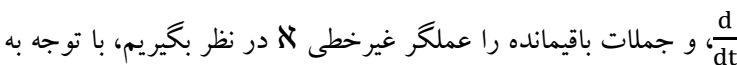

$$
\begin{aligned}
\frac{d}{d t}(\hat{x}(t))-\frac{d}{d t}\left(x_{0}\right)+p \frac{d}{d t}\left(x_{0}\right)= \\
p\left(-0.1(\hat{x}(t))+\frac{-1}{2 R}(\hat{\lambda}(t))\right)
\end{aligned}
$$

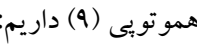

$$
\begin{aligned}
\frac{\mathrm{d}}{\mathrm{dt}}(\hat{\lambda}(\mathrm{t}))-\frac{\mathrm{d}}{\mathrm{dt}}(\alpha)+\mathrm{p} \frac{\mathrm{d}}{\mathrm{dt}}(\alpha)= \\
\quad \mathrm{p}\left(-0.2 \mathrm{Q}\left(\hat{\mathrm{x}}(\mathrm{t})-\mathrm{y}_{\text {ref }}(\mathrm{t})\right)+0.1(\hat{\lambda}(\mathrm{t}))\right)
\end{aligned}
$$

با جايگزين كردن سرىهاى (Yr) در روابط (YF) و سبس، مساوى قرادادن

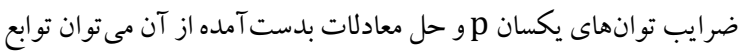

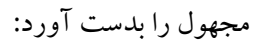

$\mathrm{x}_{1}(\mathrm{t})=\int_{\mathrm{t}_{0}}^{\mathrm{t}}\left(-0.1 \mathrm{x}_{0}+\frac{-1}{2 \mathrm{R}} \alpha\right) \mathrm{dt}$

$\mathrm{x}_{2}(\mathrm{t})=\int_{\mathrm{t}_{0}}^{\mathrm{t}}\left(-0.1 \mathrm{x}_{1}(\mathrm{t})+\frac{-1}{2 \mathrm{R}} \lambda_{1}(\mathrm{t})\right) \mathrm{dt}$

$\lambda_{1}(t)=\int_{t_{0}}^{t}-0.2 Q\left(x_{0}-y_{\text {ref }}(t)+0.1 \alpha\right) d t$

$\lambda_{2}(\mathrm{t})=\int_{\mathrm{t}_{0}}^{\mathrm{t}}\left(-0.2 \mathrm{Qx_{1 }}(\mathrm{t})+0.1 \lambda_{1}(\mathrm{t})\right) \mathrm{dt}$
آزما يشكاهى كه در بخشى از فضاى كارى بايدار و در بخش ديخر نابِيدار

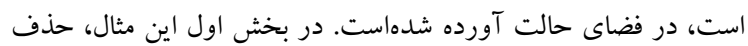
خطاى حالت ماند گار و در بخش دوم مثال، اعمال روش ييشنهادى در نقطه

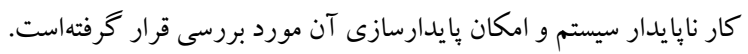

\section{1-7 كنترل يِشبين زمان ييوسته خطى نامتغير با زمان}

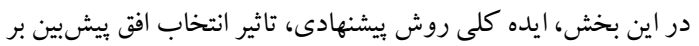

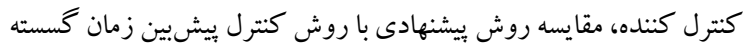
و در نهايت مقاوم بودن روش بيشنهادى به صورت عددى بررسى مى شود.

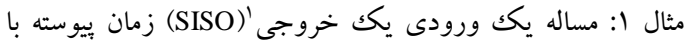
سيخنال مرجع خروجى زير رادر نظر بخيريد:

$\dot{\mathrm{x}}(\mathrm{t})=-0.1 \mathrm{x}(\mathrm{t})+\mathrm{u}(\mathrm{t})$

$\mathrm{y}(\mathrm{t})=0.1 \mathrm{x}(\mathrm{t})$

$\mathrm{y}_{\text {ref }}=0.5 \chi_{[10,20)}+0.75 \chi_{[20,30)}+0.25 \chi_{[30, \infty)}$

$\chi_{[a, b)}=\left\{\begin{array}{lr}0 & t<a \\ 1 & a \leq t<b \\ 0 & t \geq b\end{array}\right.$

كه تابع خى عبارت است از

هدف طر احى كنترل كننده بيشيين براى دنبال كردن سيكنال مرجع بوسيله

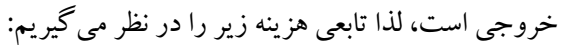
$J=\overbrace{\left[y\left(t_{f}\right)-y_{\text {ref }}\left(t_{f}\right)\right]^{T} H\left[y\left(t_{f}\right)-y_{\text {ref }}\left(t_{f}\right)\right]}$

$$
\begin{aligned}
& +\int_{\mathrm{t}_{0}}^{\mathrm{t}_{\mathrm{f}}}\left[\mathrm{y}(\mathrm{t})-\mathrm{y}_{\text {ref }}(\mathrm{t})\right]^{\mathrm{T}} \mathrm{Q}[\mathrm{y}(\mathrm{t}) \\
& \left.-\mathrm{y}_{\text {ref }}(\mathrm{t})\right]+\mathrm{u}^{\mathrm{T}} \mathrm{Rudt}
\end{aligned}
$$

طبق روش بيشنهادى، براى تشكيل دستگاه معادلات ديفر انسيل-جبرى با

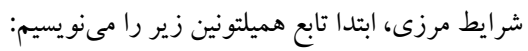
$\mathcal{H}(\mathrm{x}(\mathrm{t}), \mathrm{u}(\mathrm{t}), \lambda(\mathrm{t}), \mathrm{t}) \triangleq \mathrm{Q}\left(0.1 \mathrm{x}(\mathrm{t})-\mathrm{y}_{\text {ref }}(\mathrm{t})\right)^{2}+$ $\left.\mathrm{Ru}^{2}(\mathrm{t})+\lambda^{\mathrm{T}}(\mathrm{t})[-0.1 \mathrm{x}(\mathrm{t})+\mathrm{u}(\mathrm{t}))\right]$

با مشتق گيرى از اين تابع نسبت به U خواهيم داشت: $\frac{\partial \mathcal{H}}{\partial \mathrm{u}}=2 \mathrm{Ru}+\lambda(\mathrm{t})=0 \Rightarrow \mathrm{u}=\frac{-\lambda(\mathrm{t})}{2 \mathrm{R}}$

Such that $\frac{\partial^{2} \mathcal{H}}{\partial \mathrm{u}^{2}}=2 \mathrm{R}>0, R:$ ماتريس (اسكالر) معين مثبت به اين ترتيب، دستخاه معادلات ديفرانسيل با شرايط مرزى معادل مساله كنترل بهينه بر بازه بروزرسانى $\left\{\begin{array}{l}\dot{\mathrm{x}}(\mathrm{t})=-0.1 \mathrm{x}(\mathrm{t})+\frac{-\lambda(\mathrm{t})}{2 \mathrm{R}} \\ \dot{\lambda}(\mathrm{t})=-2 \mathrm{Q}\left(0.1 \mathrm{x}(\mathrm{t})-\mathrm{y}_{\text {ref }}(\mathrm{t})\right) \cdot(0.1)+0.1 \lambda(\mathrm{t}) \\ \mathrm{x}\left(\mathrm{t}_{0}\right)=\mathrm{x}_{0} \\ \left.\frac{\partial \mathrm{h}}{\partial \mathrm{x}}\right|_{\mathrm{t}=\mathrm{t}_{\mathrm{f}}}=2 \mathrm{H}\left(0.1 \mathrm{x}\left(\mathrm{t}_{\mathrm{f}}\right)-\mathrm{y}_{\mathrm{ref}}\left(\mathrm{t}_{\mathrm{f}}\right)\right) \cdot(0.1)-\lambda\left(\mathrm{t}_{\mathrm{f}}\right)=0\end{array}\right.$

(YI) 

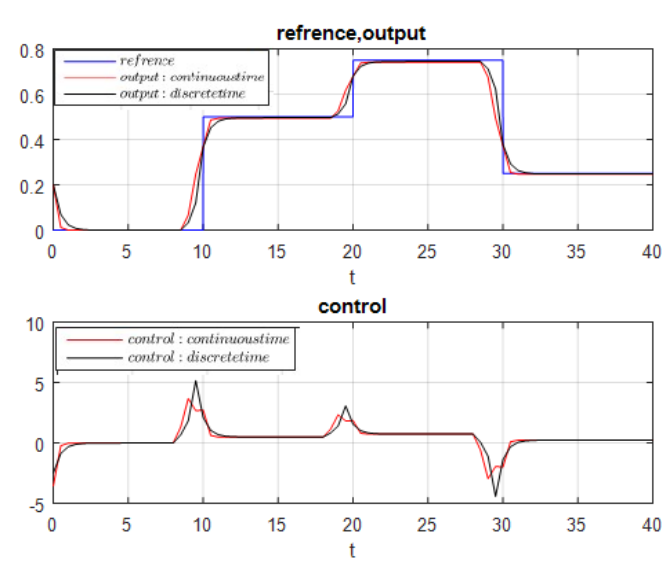

شكل Y): مقايسه هاسخ سيستم مثال ( به دو روش كنترل بيشبين زمان HP = بيوسته و زمان گسسته با افق ييشبين 3

جدول ا: نتايج مقايسه كنترل بيشبين بيوسته و گسسته مثال 1

\begin{tabular}{|c|c|c|c|c|}
\hline يله سوم & يله دوم & بֶله اول & \multicolumn{2}{|c|}{ معيار } \\
\hline r/aV & $r / T \Delta$ & T/YY & بيوسته & زمان \\
\hline $9 / 0$ & $r / \Delta r$ & $4 / 19$ & گ كسته & ( \\
\hline $1 / 99 \mathrm{ra}$ & $|/ F| \wedge \Delta$ & $1 / \Delta F \cdot V$ & ته به بيوسته & كنترل ز \\
\hline
\end{tabular}

Q با توجه به Ts =

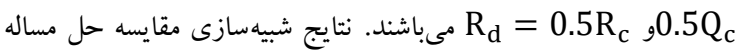

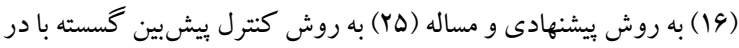
نظر گرفتن جدول(1) آورده شدهاست. مشاهده مىشود كه زمان نشست و دامنه

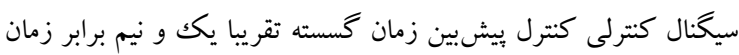

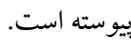
در الكوريتم يِينهادى، مقدار افق بيش بين در نظر كرفته شده فقط در

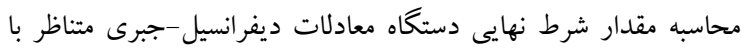

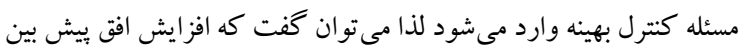

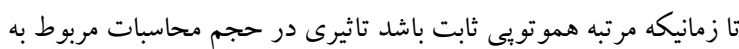

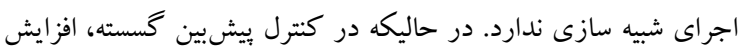

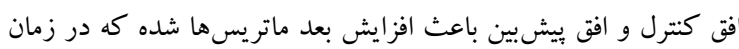

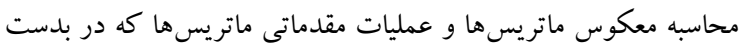

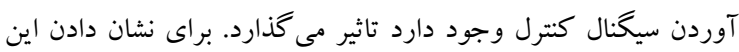

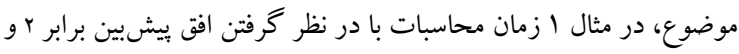

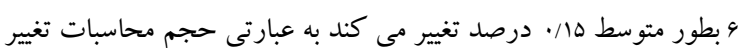

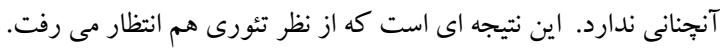

با حل معادلات فوق، توابع

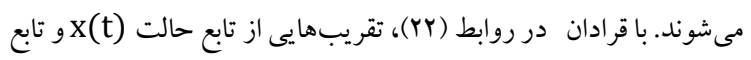

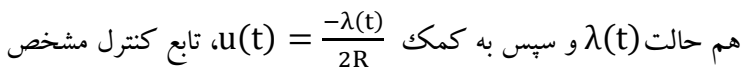
مىشود. نتايج شبيهسازى با درد نظرگرفتن پِارامترهاى مساله به صورت

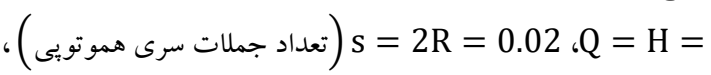

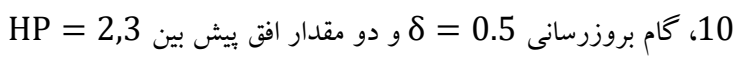

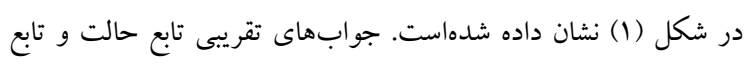

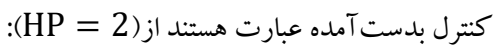

$x(t)=\left\{\begin{array}{cc}2-3.3148 t & {[0,0.5]} \\ 0.6266-0.5679 t & {[0.5,1]} \\ 0.1560-0.0973 t & {[1,1.5]} \\ 0.0351-0.0167 t & {[1.5,2]} \\ \vdots & \end{array}\right.$

$u(t)=\left\{\begin{array}{cc}9.688 t-3.1147 & {[0,0.5]} \\ 1.6598 t-1.3635 & {[0.5,1]} \\ 0.2843 t-0.3757 & {[1,1.5]} \\ 0.04871 t-0.0887 & {[1.5,2]} \\ \vdots & \end{array}\right.$

همانطور كه در شكل (1) مشاهده مىشود، كنترل كننده بيشبين،

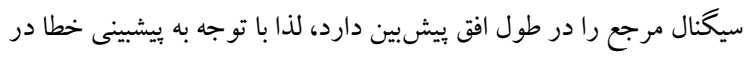

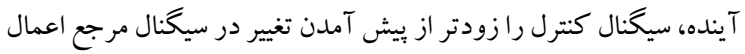
مى كند. براى مقايسه روش بيشنهادى با كنترل بيشبين زمان گسسته [rr]، ابتدا

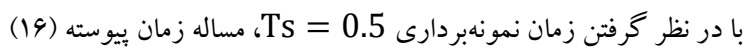
را به سيستم زمان گسسته تبديل مى كنيم: بطون

$\left\{\begin{array}{l}x(k+1)=0.9512 x(k)+0.4877 u(k) \\ y(k)=0.1 x(k)\end{array}\right.$
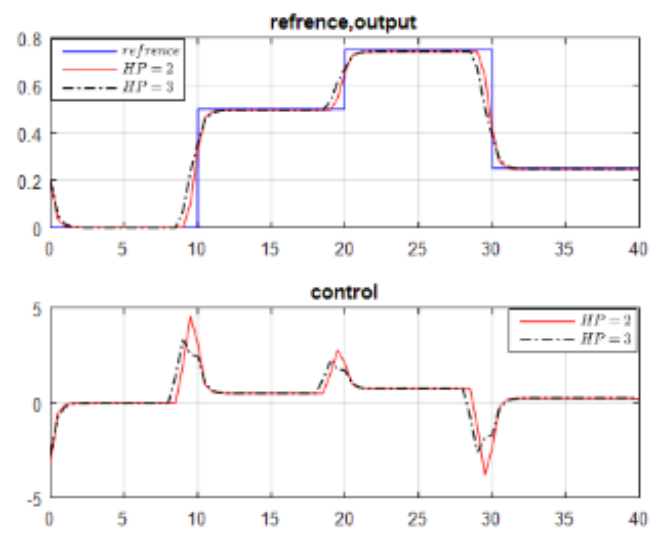

شكل () ياسخ سيستم كنترل ييشبين زمان ييوسته مثال ا، براى دو مقدار افق ييشبين 2,3 = 
سيخنال مرجع بوسيله خروجى به روش كترل بِيشبين زمان بيوسته

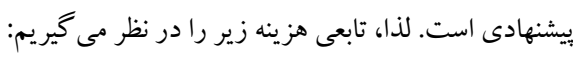

$$
\begin{aligned}
& J=\overbrace{\left[y\left(t_{f}\right)-y_{\text {ref }}\left(t_{f}\right)\right]^{T}\left[\begin{array}{cc}
\mathrm{H}_{1} & 0 \\
0 & H_{2}
\end{array}\right]\left[y\left(t_{f}\right)-y_{\text {ref }}\left(t_{f}\right)\right]}^{h} \\
& +\int_{\mathrm{t}_{0}}^{\mathrm{t}_{\mathrm{f}}}[\mathrm{y}(\mathrm{t}) \\
& \left.-\mathbf{y}_{\text {ref }}(\mathrm{t})\right]^{\mathrm{T}}\left[\begin{array}{cc}
\mathrm{Q}_{1} & 0 \\
0 & \mathrm{Q}_{2}
\end{array}\right][\mathbf{y}(\mathrm{t}) \\
& \left.-\mathbf{y}_{\text {ref }}(\mathrm{t})\right]+\mathbf{u}^{\mathrm{T}}\left[\begin{array}{cc}
\mathrm{R}_{1} & 0 \\
0 & \mathrm{R}_{2}
\end{array}\right] \mathbf{u d t}
\end{aligned}
$$
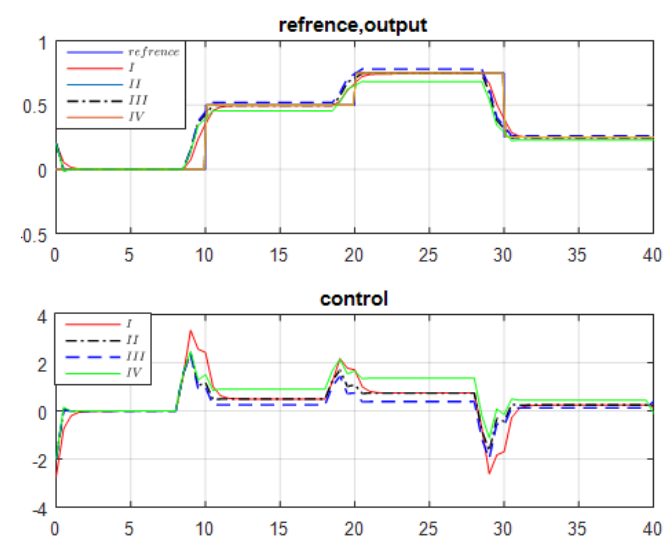

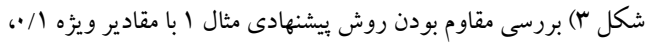

$$
\therefore / r, \%
$$

بتدا تابع هميلتونين را تشكيل مىدهيم:

$$
\mathcal{H}(\mathbf{x}(\mathrm{t}), \mathbf{u}(\mathrm{t}), \lambda(\mathrm{t}), \mathrm{t}) \triangleq[\mathbf{y}(\mathrm{t})-
$$

$$
\begin{array}{r}
\left.\mathbf{y}_{\text {ref }}(\mathrm{t})\right]^{\mathrm{T}}\left[\begin{array}{cc}
Q_{1} & 0 \\
0 & Q_{2}
\end{array}\right]\left[\mathbf{y}(\mathrm{t})-\mathbf{y}_{\text {ref }}(\mathrm{t})\right]+ \\
\mathbf{u}^{\mathrm{T}}\left[\begin{array}{cc}
R_{1} & 0 \\
0 & R_{2}
\end{array}\right] \mathbf{u}+\lambda^{\mathrm{T}}(\mathrm{t}) \mathrm{f}(\mathbf{x}, \mathbf{u}, \mathrm{t})
\end{array}
$$

$$
\text { با مشتق گيرى از آن نسبت به U داريم: }
$$

$$
\begin{aligned}
\frac{\partial \mathcal{H}}{\partial \mathbf{u}}=2\left[\begin{array}{cc}
R_{1} & 0 \\
0 & R_{2}
\end{array}\right] \mathbf{u}+\boldsymbol{\lambda}^{\mathrm{T}}(\mathrm{t}) \frac{\partial \mathrm{f}(\mathbf{x}, \mathbf{u}, \mathrm{t})}{\partial \mathbf{u}}=\mathbf{0} \\
\\
\Rightarrow\left\{\begin{array}{c}
\mathrm{u}_{1}=\frac{-0.5}{2 \mathrm{R}_{1}} \lambda_{1}(\mathrm{t}) \\
\mathrm{u}_{2}=\frac{-(2+\sin \mathrm{t})}{2 \mathrm{R}_{2}} \lambda_{2}(\mathrm{t})
\end{array}\right.
\end{aligned}
$$

such that $\frac{\partial^{2} \mathcal{H}}{\partial \mathbf{u}^{2}}=2\left[\begin{array}{cc}R_{1} & 0 \\ 0 & R_{2}\end{array}\right]$

$$
\text { ماتريس معين مثبت : }
$$

به اين ترتيب، دستگاه معادلات ديفرانسيل با شرايط مرزى مساله عبارت
با توجه به وجود اغتشاشات نامعلوم و عدم تطابق دقيق مدل و يلنت، بحث

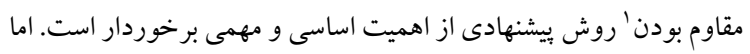

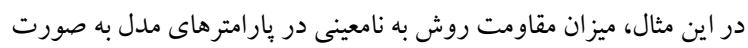

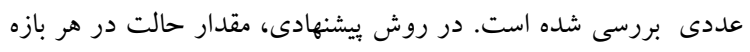

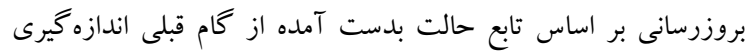

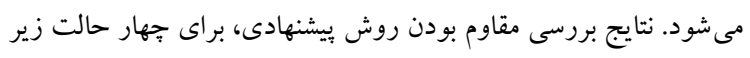
در شكل (r) مشاهده مى شود:

اندازه إنيرى حالت سيستم در هر بازه بروزرسانى بر اساس تابع

$$
\text { حالت بدست آمده از حل مساله. }
$$

اندازهگيرى حالت سيستم در هر بازه بروزرسانى بر اساس ماسل اعمال سيخنال كنترل بدست آمده بر مدل (19).

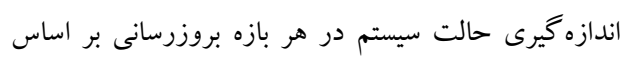
III

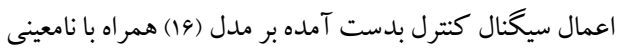
كه مقدار ويزه آن هـ / •-- درنظر گرفته شده است.

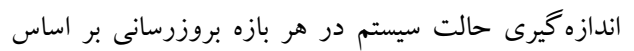
IV

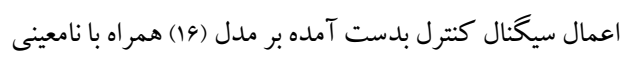
كه مقدار ويزه آن ب/ • - درنظر كرفته شده است.

همانطور كه در شكل (r) مشاهده مىشود، على رغم اينكه سرعت يلنت

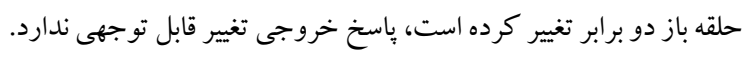

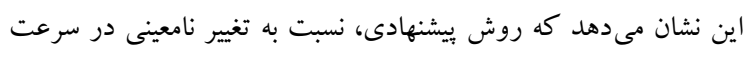
يلنت حلقه باز مقاومت خوبى دارد.

r-7 كنترل ييشبين زمان وييوسته خطى متغير با زمان

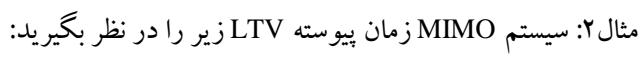
$\dot{\mathbf{x}}(\mathrm{t})=\mathrm{f}(\mathbf{x}, \mathbf{u}, \mathrm{t})$

$$
\begin{aligned}
& =\left[\begin{array}{cc}
0.25 \sin t+0.5 & 0 \\
0 & -0.25 \sin 2 t-0.5
\end{array}\right] \mathbf{x}(\mathrm{t}) \\
& +\left[\begin{array}{cc}
0.5 & 0 \\
0 & 2+\sin t
\end{array}\right] \mathbf{u}(\mathrm{t}) \\
& \mathbf{y}(\mathrm{t})=\left[\begin{array}{cc}
1 & 0 \\
-1 & -1
\end{array}\right] \mathbf{x}(\mathrm{t})
\end{aligned}
$$
مقادير ويزه سيستم عبار تند از :

$\{0.25 \sin t+0.5,-0.25 \sin 2 t-0.5 \mid t \in \mathbb{R}\}$

واضح است كه مقادير ويزه در بازهاى $[0.25,0.75]$ ك] فرض كنيد حالت اوليه سيستم

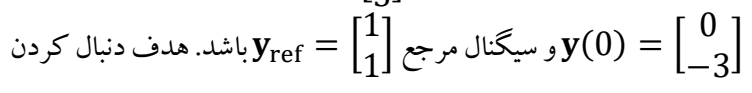

\section{' Robustness}




$$
\begin{aligned}
& \left(\dot{\mathrm{x}}_{1}(\mathrm{t})=(0.25 \sin \mathrm{t}+0.5) \mathrm{x}_{1}(\mathrm{t})+0.5\left(\frac{-0.5}{2 \mathrm{R}_{1}} \lambda_{1}(\mathrm{t})\right)\right. \\
& \dot{\mathrm{x}}_{2}(\mathrm{t})=(-0.25 \sin 2 \mathrm{t}-0.5) \mathrm{x}_{2}(\mathrm{t})+(2+\sin \mathrm{t})\left(\frac{-(2+\sin \mathrm{t})}{2 \mathrm{R}_{2}} \lambda_{2}(\mathrm{t})\right) \\
& \dot{\lambda_{1}}(\mathrm{t})=-2 \mathrm{Q}_{1}\left(\mathrm{x}_{1}(\mathrm{t})-\mathrm{y}_{1 \text { ref }}(\mathrm{t})\right)+2 \mathrm{Q}_{2}\left(-\mathrm{x}_{1}(\mathrm{t})-\mathrm{x}_{2}(\mathrm{t})-\mathrm{y}_{2 \text { ref }}(\mathrm{t})\right)-0.25 \sin t \lambda_{1}(\mathrm{t})-0.5 \lambda_{1}(\mathrm{t}) \\
& \dot{\lambda_{2}}(\mathrm{t})=2 \mathrm{Q}_{2}\left(-\mathrm{x}_{1}(\mathrm{t})-\mathrm{x}_{2}(\mathrm{t})-\mathrm{y}_{2 \operatorname{ref}}(\mathrm{t})\right)+0.25 \sin 2 \mathrm{t} \lambda_{2}(\mathrm{t})+0.5 \lambda_{2}(\mathrm{t}) \\
& \mathbf{x}\left(\mathrm{t}_{0}\right)=\mathbf{x}_{\mathbf{0}} \\
& \left.\frac{\partial \mathrm{h}}{\partial \mathrm{x}_{1}}\right|_{\mathrm{t}=\mathrm{t}_{\mathrm{f}}}=2 \mathrm{H}_{1}\left(\mathrm{x}_{1}\left(\mathrm{t}_{\mathrm{f}}\right)-\mathrm{y}_{1 \mathrm{ref}}\left(\mathrm{t}_{\mathrm{f}}\right)\right)-2 \mathrm{H}_{2}\left(-\mathrm{x}_{1}\left(\mathrm{t}_{\mathrm{f}}\right)-\mathrm{x}_{2}\left(\mathrm{t}_{\mathrm{f}}\right)-\mathrm{y}_{2 \mathrm{ref}}\left(\mathrm{t}_{\mathrm{f}}\right)\right)-\lambda_{1}\left(\mathrm{t}_{\mathrm{f}}\right)=0 \\
& \begin{array}{l}
\left.\frac{\partial \mathrm{h}}{\partial \mathrm{x}_{2}}\right|_{\mathrm{t}=\mathrm{t}_{\mathrm{f}}}=-2 \mathrm{H}_{2}\left(-\mathrm{x}_{1}\left(\mathrm{t}_{\mathrm{f}}\right)-\mathrm{x}_{2}\left(\mathrm{t}_{\mathrm{f}}\right)-\mathrm{y}_{2 \mathrm{ref}}\left(\mathrm{t}_{\mathrm{f}}\right)\right)-\lambda_{2}\left(\mathrm{t}_{\mathrm{f}}\right)=0 \\
\lambda\left(\mathrm{t}_{0}\right)=\boldsymbol{\alpha}
\end{array} \\
& \left\{\begin{array}{c}
\hat{\mathbf{x}}\left(\mathrm{t}_{0}\right)=\mathbf{x}_{\mathbf{0}}, \quad \text { if } \mathbf{x}_{\mathbf{0}}(\mathbf{t})=\mathbf{x}_{\mathbf{0}} \\
\text { then } \mathbf{x}_{\mathbf{1}}\left(\mathbf{t}_{\mathbf{0}}\right)=\cdots=\mathbf{x}_{\mathbf{s}}\left(\mathbf{t}_{\mathbf{0}}\right)=\mathbf{0} \\
\hat{\lambda}\left(\mathrm{t}_{0}\right)=\boldsymbol{\alpha}, \quad \text { if } \boldsymbol{\lambda}_{\mathbf{0}}(\mathbf{t})=\boldsymbol{\alpha} \\
\text { then } \boldsymbol{\lambda}_{\mathbf{1}}\left(\mathbf{t}_{\mathbf{0}}\right)=\cdots=\boldsymbol{\lambda}_{\mathbf{s}}\left(\mathbf{t}_{\mathbf{0}}\right)=\mathbf{0}
\end{array}\right. \\
& \text { جواب نيمه تحليلى مر تبه S دستكاه (·r)، طبق روش اختلال همو توبى به } \\
& \text { صورت زيردر نظر كرفته مى شود: }
\end{aligned}
$$

آنغاه با توجه به هموتويى(9) داريم:

$$
\begin{aligned}
& \int \frac{\mathrm{d}}{\mathrm{dt}}\left(\hat{\mathrm{x}}_{1}(\mathrm{t})\right)-\frac{\mathrm{d}}{\mathrm{dt}}\left(\mathrm{x}_{01}\right)+\mathrm{p} \frac{\mathrm{d}}{\mathrm{dt}}\left(\mathrm{x}_{01}\right)=\mathrm{p}\left(\left(0.25 \mathrm{~g}_{1}+0.5\right) \hat{\mathrm{x}}_{1}(\mathrm{t})+0.5\left(\frac{-0.5}{2 \mathrm{R}_{1}} \hat{\lambda}_{1}(\mathrm{t})\right)\right) \\
& \frac{\mathrm{d}}{\mathrm{dt}}\left(\hat{\mathrm{x}}_{2}(\mathrm{t})\right)-\frac{\mathrm{d}}{\mathrm{dt}}\left(\mathrm{x}_{02}\right)+\mathrm{p} \frac{\mathrm{d}}{\mathrm{dt}}\left(\mathrm{x}_{02}\right)=\mathrm{p}\left(\begin{array}{c}
\left(-0.25 \mathrm{~g}_{2}-0.5\right) \hat{\mathrm{x}}_{2}(\mathrm{t})+ \\
\left(2+\mathrm{g}_{1}\right)\left(\frac{-\left(2+\mathrm{g}_{1}\right)}{2 \mathrm{R}_{2}} \hat{\lambda}_{2}(\mathrm{t})\right)
\end{array}\right) \\
& \frac{\mathrm{d}}{\mathrm{dt}}\left(\hat{\lambda}_{1}(\mathrm{t})\right)-\frac{\mathrm{d}}{\mathrm{dt}}\left(\alpha_{1}\right)+\mathrm{p} \frac{\mathrm{d}}{\mathrm{dt}}\left(\alpha_{1}\right)=\mathrm{p}\left(\begin{array}{c}
-2 \mathrm{Q}_{1}\left(\hat{\mathrm{x}}_{1}(\mathrm{t})-\mathrm{y}_{1 \mathrm{ref}}(\mathrm{t})\right)+ \\
2 \mathrm{Q}_{2}\left(-\hat{\mathrm{x}}_{1}(\mathrm{t})-\hat{\mathrm{x}}_{2}(\mathrm{t})-\mathrm{y}_{2 \mathrm{ref}}(\mathrm{t})\right)- \\
0.25 \mathrm{~g}_{1} \hat{\lambda}_{1}(\mathrm{t})-0.5 \hat{\lambda}_{1}(\mathrm{t})
\end{array}\right) \\
& \frac{\mathrm{d}}{\mathrm{dt}}\left(\hat{\lambda}_{2}(\mathrm{t})\right)-\frac{\mathrm{d}}{\mathrm{dt}}\left(\alpha_{2}\right)+\mathrm{p} \frac{\mathrm{d}}{\mathrm{dt}}\left(\alpha_{2}\right)=\mathrm{p}\left(\begin{array}{c}
2 \mathrm{Q}_{2}\left(-\hat{\mathrm{x}}_{1}(\mathrm{t})-\hat{\mathrm{x}}_{2}(\mathrm{t})-\mathrm{y}_{2 \mathrm{ref}}(\mathrm{t})\right)+ \\
0.25 \mathrm{~g}_{2} \hat{\lambda}_{2}(\mathrm{t})+0.5 \hat{\lambda}_{2}(\mathrm{t})
\end{array}\right)
\end{aligned}
$$

S = نتايج شبيه سازى با در نظر كرفتن بارامترهاى مساله به صورت

(تعداد جملات سرى هموتويى)،

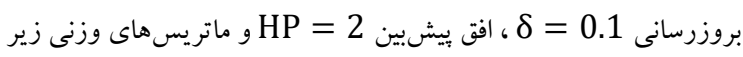

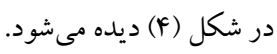

I. $\quad \mathrm{R}=\left[\begin{array}{cc}0.1 & 0 \\ 0 & 0.1\end{array}\right], \mathrm{Q}=\left[\begin{array}{cc}10 & 0 \\ 0 & 2\end{array}\right], \mathrm{H}=\left[\begin{array}{cc}10 & 0 \\ 0 & 1\end{array}\right]$ 
11

طراحى كتترل كننده هيشبين غير خطى زمان بيوسته با حل دستكاه معادلات ديفرانسيل - جبرى با شرايط مرزى به روش اختلال هموتويى

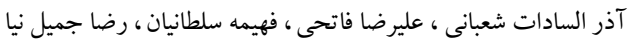

جو اببهاى تقريبى تابع حالت و تابع كنترل بدست آمده براى انتخاب ماتريس هاى وزنى (I) عبارت هستند از: تكرئ
II. $\mathrm{R}=\left[\begin{array}{ll}1 & 0 \\ 0 & 1\end{array}\right], \mathrm{Q}=\left[\begin{array}{cc}10 & 0 \\ 0 & 2\end{array}\right], \mathrm{H}=\left[\begin{array}{cc}10 & 0 \\ 0 & 1\end{array}\right]$

III. $\mathrm{R}=\left[\begin{array}{ll}1 & 0 \\ 0 & 1\end{array}\right], \mathrm{Q}=\left[\begin{array}{cc}20 & 0 \\ 0 & 5\end{array}\right], \mathrm{H}=\left[\begin{array}{cc}20 & 0 \\ 0 & 2\end{array}\right]$

$x_{1}(t)=\left\{\begin{array}{c}4.5900 t-2.5000 t^{2}+0.1912 t^{3} \\ 0.0726+4.0445 t-4.2969 t^{2}+0.1508 t^{3}-0.0036 t^{4}-0.0002 t^{5} \\ \vdots\end{array}\right.$

$x_{2}(t)=\left\{\begin{array}{c}3-48.1927 t+136.2786 t^{2}+103.1506 t^{3}+19.1210 t^{4}-0.1946 t^{5} \\ 1.5171-22.1763 t+32.1109 t^{2}+27.5618 t^{3}+4.0592 t^{4}-0.5013 t^{5}+ \\ 0.0155 t^{6}-.0006 t^{7} \\ \vdots\end{array}\right.$

$u_{1}(t)=\left\{\begin{array}{cc}9.1799-14.5900 t-100.7648 t^{2}-78.9141 t^{3}-9.6559 t^{4} & {[0,0.1]} \\ 8.2591-26.3414 t+31.8454 t^{2}-19.6921 t^{3}-2.3895 t^{4}+0.1688 t^{5}-0.0028 t^{5} & {[0.1,0.2]}\end{array}\right.$

$u_{2}(t)=\left\{\begin{array}{l}-23.3464+136.6536 t-766.6469 t^{2}-717.9417 t^{3}-188.4085 t^{4}-19.8201 t^{5}[0,0.1] \\ -12.9709+75.4939 t-158.1542 t^{2}-185.4210 t^{3}-47.3085 t^{4}-2.4951 t^{5}+ \\ 0.6232 t^{6}-0.0262 t^{7}+0.0004 t^{8} \\ {[0.1,0.2]}\end{array}\right.$

در اين بخش هدف، كنترل مسير مجزاى SISO، افقى است. در عمل با بستن

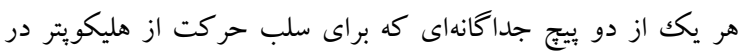

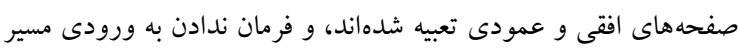

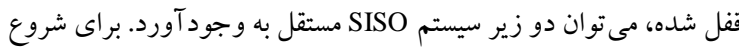
طراحى كنترل كننده، ابتدا، معادلات ديفرانسيل غير سطى في فضاى حالت حاكم بر هليكويتر را در نظر مى گيريم: $\mathrm{f}_{\Psi}\left(\mathrm{x}(\mathrm{t}), \mathrm{u}_{1}(\mathrm{t}), \mathrm{t}\right)$

$=\left[\begin{array}{c}\frac{1}{\mathrm{I}}\left(-\tau_{\mathrm{g}} \sin \left(\mathrm{x}_{1}\right)-\mathrm{B}_{\varphi} \mathrm{x}_{2}+\mathrm{a}_{1} \mathrm{x}_{3}^{2}+\mathrm{b}_{1} \mathrm{x}_{3}\right) \\ \mathrm{x}_{4} \\ \frac{1}{\mathrm{~T}_{1}^{2}}\left(\mathrm{u}_{1}-\mathrm{x}_{3}-2 \mathrm{~T}_{1} \mathrm{x}_{4}\right)\end{array}\right]$

$\mathbf{x}(\mathrm{t})=\left[\begin{array}{c}\mathrm{x}_{1} \\ \mathrm{x}_{2} \\ \mathrm{x}_{3} \\ \mathrm{x}_{4}\end{array}\right]=\left[\begin{array}{c}\psi \\ \dot{\psi} \\ \alpha \\ \dot{\alpha}\end{array}\right]$

$g_{\psi}\left(x(t), u_{1}(t), t\right)=\left[x_{1}\right]$

در مدل ديناميكى بيانشده، جهارمتغير حالت وجود دارد كه حالت و

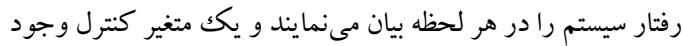

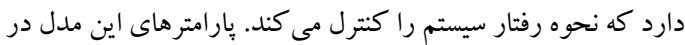

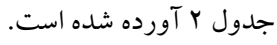

جدول r: يار امترهاى فضاى حالت سيستم ديناميكى مثال (r)

\begin{tabular}{|c|c|c|}
\hline$a_{1}=0.1807$ & $I=0.0054$ & $T_{1}=0.3$ \\
\hline$b_{1}=0.0028$ & $B_{\varphi}=0.0011$ & $\boldsymbol{\tau}_{\mathbf{g}}=\mathbf{0 . 0 7 5 5}$ \\
\hline
\end{tabular}

از آنجايى كه تابعى هزينه بر اساس متغيرهاى حالت نوشته مىشود، براى

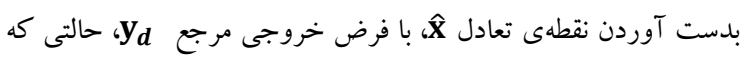

با توجه به نتايج شكل (F)، ديده مىشود كه با تنظيم ماتريسهاى وزنى

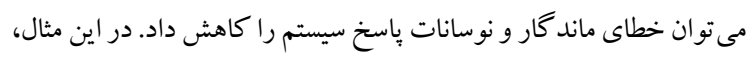
بهترين باسخ در انتخاب هاى ماتريسهاى وزنى فوق، انتخاب (I) است كه خروجى اول تقريبا بدون نوسان سيخنال مرجع را دنبال مى كند.
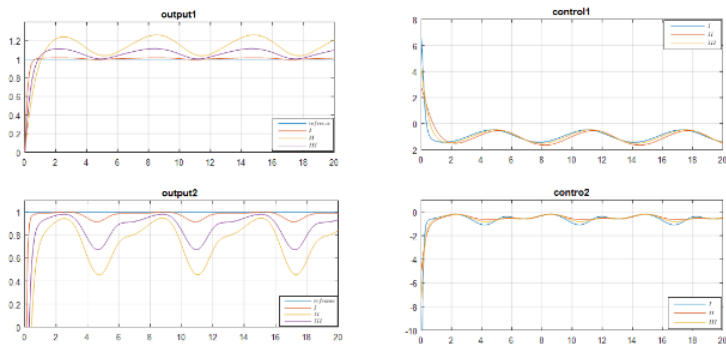

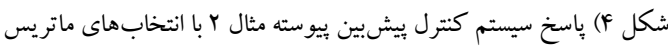

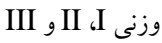

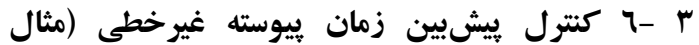

[YD ] CE150 كاربردى هليكوڤتر مدل

در اين بخش عملكرد روش بيشنهادى براى طراحى كنترلكننده

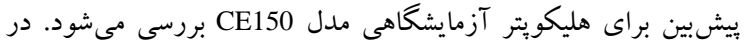

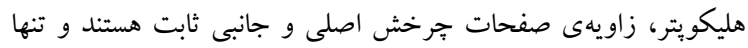

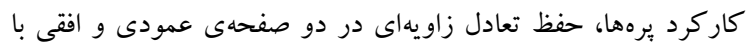
مشخصههاى زاويهاى ل و \$ مىباشد. تنها ورودىهاى كنترلى هليكويتر

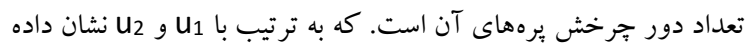

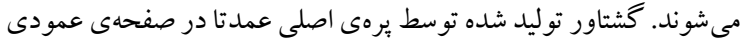
Elevation در صفحهى افقى Azimuth (\$) تاثير دارد. با بررسى شرايط كنترليذيرى و رويتيذيرى دو زير سيستم SISO افقى و عمودى مطمئن مى شويم كه هر دو قابل كنترل هستند. 
(广人)

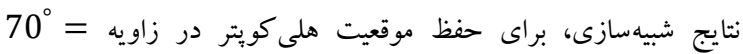
1.2217 (rad)

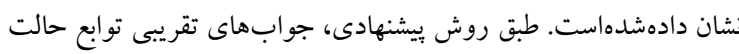

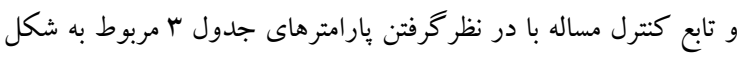

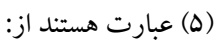

$$
\mathrm{x}_{1}(\mathrm{t})
$$$$
=\left\{\begin{array}{c}
1.0472+0.3153 \mathrm{t}^{2} \\
1.0472+0.0017 \mathrm{t}+0.2966 \mathrm{t}^{2} \\
\vdots
\end{array}\right.
$$

$\mathrm{x}_{2}(\mathrm{t})$

$=\left\{\begin{array}{c}0.6306 \mathrm{t}-0.0642 \mathrm{t}^{2} \\ 0.0003+0.6481 \mathrm{t}-0.5495 \mathrm{t}^{2} \\ \vdots\end{array}\right.$

$[0,0.05]$

$[0.05,0.1]$

$\mathrm{x}_{3}(\mathrm{t})$

$=\left\{\begin{array}{c}0.6093-0.2476 \mathrm{t}^{2} \\ 0.6095-0.0138 \mathrm{t}-0.0462 \mathrm{t}^{2} \\ \vdots\end{array}\right.$

$[0,0.5]$

$[0.05,0.1]$

$\mathrm{x}_{4}(\mathrm{t})$

$=\left\{\begin{array}{c}-0.4952+2.5437 \mathrm{t}^{2} \\ -0.0080-0.3240 \mathrm{t}+2.3161 \mathrm{t}^{2} \\ \vdots\end{array}\right.$

$[0,0.05]$

$[0.05,0.1]$

$\mathrm{u}(\mathrm{t})$

$=\left\{\begin{array}{c}3.6041-97.4538 \mathrm{t}+614.1250 \mathrm{t}^{2}[0,0.05] \\ 9.8578-155.3022 \mathrm{t}+597.2224 \mathrm{t}^{2}[0.05,0.1] \\ \vdots\end{array}\right.$

همانطوركه در شكل(ه) ديده مىشود، با شروع از نقطه

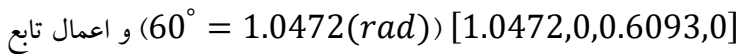

كتترل بدست آمده بر هليكويتر، سيستم، مسير مرجع را با خطاى حالت

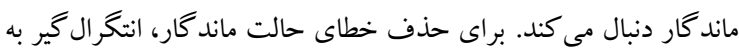
مساله اضافه مى كنيم. با اين كار تعداد حالتهاى سيستم ب ب تا اضافه مى شود.

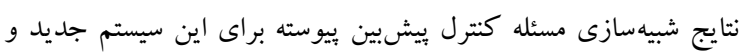
تنظيم مجدد بارامترهاى ماتريس هاى وزنى، در شكل (9) آورده شدهاست.

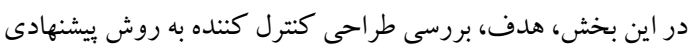
براى يكك سيستم نايايدار و امكان يايدارسازى آن است. از آنجايى كه

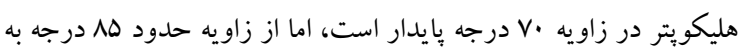

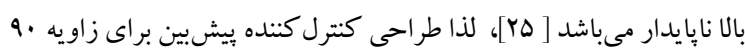

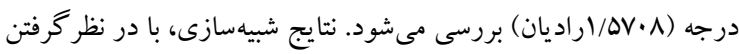

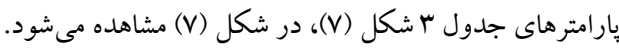

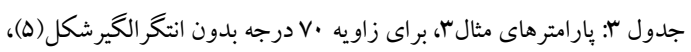

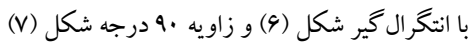

\begin{tabular}{|c|c|c|c|}
\hline ي پِارامترها & شكل (ه) & شكل (9) & شكل(v) \\
\hline $\mathrm{HP}$ & 2 & 2 & 2 \\
\hline S & 3 & 3 & 3 \\
\hline $\bar{\delta}$ & 0.05 & 0.1 & 0.1 \\
\hline $\mathrm{R}$ & 1 & 1 & 0.25 \\
\hline $\mathrm{H}_{1}=\mathrm{H}_{3}$ & 8 & 8 & $0.25,1,10,20$ \\
\hline$Q_{1}=Q_{3}$ & 80 & 80 & 10 \\
\hline
\end{tabular}

هليكويتر مستقيما رو به جلو و با زاويهى $\mathbf{~}$ قرار داشته باشد، معادلات زير

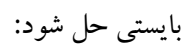

$$
\left\{\begin{aligned}
\dot{x}(t) & =f(x(t), u(t), t)=0 \\
y_{d} & =r
\end{aligned}\right.
$$

با حل معادلات فوق نقطه تعادل بدست مى آيد:

if $\mathrm{y}_{d}=70^{0}=0.9397$ (radian) then $\hat{\mathrm{x}}$

$=[0.9397,0,0.6189,0]^{\mathrm{T}}$ and $\widehat{\mathbf{u}}=[0.6189]$

if $\mathrm{y}_{d}=90^{\circ}=1.5708$ (radian) then $\hat{\mathrm{x}}$ $=[1.5708,0,0.6387,0]^{\mathrm{T}}$ and $\hat{\mathrm{u}}=[0.6387]$ هدف حفظ هليكويتر در نقطه تعادل است، لذا براى طراحى كنترل كننده،

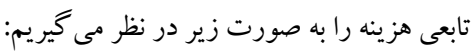

$\min \mathrm{J}=\left[\mathrm{x}_{1}\left(\mathrm{t}_{\mathrm{f}}\right)-\mathrm{x}_{1 \mathrm{ref}}\left(\mathrm{t}_{\mathrm{f}}\right)\right]^{\mathrm{T}} \mathrm{H}_{1}\left[\mathrm{x}_{1}\left(\mathrm{t}_{\mathrm{f}}\right)-\right.$

$\left.\mathrm{x}_{1 \text { ref }}\left(\mathrm{t}_{\mathrm{f}}\right)\right]+\left[\mathrm{x}_{3}\left(\mathrm{t}_{\mathrm{f}}\right)-\mathrm{x}_{3 \mathrm{ref}}\left(\mathrm{t}_{\mathrm{f}}\right)\right]^{\mathrm{T}} \mathrm{H}_{3}\left[\mathrm{x}_{3}\left(\mathrm{t}_{\mathrm{f}}\right)-\right.$

$\left.\mathrm{x}_{3 \mathrm{ref}}\left(\mathrm{t}_{\mathrm{f}}\right)\right]+\int_{\mathrm{t}_{0}}^{\mathrm{t}_{\mathrm{f}}}\left[\mathrm{x}_{1}(\mathrm{t})-\mathrm{x}_{1 \mathrm{ref}}(\mathrm{t})\right]^{\mathrm{T}} \mathrm{Q}_{1}\left[\mathrm{x}_{1}(\mathrm{t})-\right.$

$\left.\mathrm{x}_{1 \text { ref }}(\mathrm{t})\right]+\left[\mathrm{x}_{3}(\mathrm{t})-\mathrm{x}_{3 \text { ref }}(\mathrm{t})\right]^{\mathrm{T}} \mathrm{Q}_{3}\left[\mathrm{x}_{3}(\mathrm{t})-\right.$

$\left.\mathrm{x}_{3 \mathrm{ref}}(\mathrm{t})\right]+\mathrm{u}^{\mathrm{T}} \mathrm{Rudt}$

(r4)

طبق الكوريتم r، با حل دستكاه معادلات ديفرانسيل با شرايط مرزى زير با روش اختلال هموتويى در هر زير بازه بروزرسانى، تابع حالت و تابع كنترل بهينه بدستمى آيند: $\left\{\begin{array}{l}\dot{\mathrm{x}}_{1}=\mathrm{x}_{2} \\ \dot{\mathrm{x}}_{2}=\frac{1}{\mathrm{I}}\left(-\tau_{\mathrm{g}} \sin \left(\mathrm{x}_{1}\right)-\mathrm{B}_{\emptyset} \mathrm{x}_{2}+\mathrm{a}_{1} \mathrm{x}_{3}^{2}+\mathrm{b}_{1} \mathrm{x}_{3}\right) \\ \dot{\mathrm{x}}_{3}=\mathrm{x}_{4} \\ \dot{\mathrm{x}}_{4}=\frac{1}{\mathrm{~T}_{1}^{2}}\left(-\mathrm{x}_{3}-2 \mathrm{~T}_{1} \mathrm{x}_{4}-\frac{1}{\mathrm{~T}_{1}^{2} 2 \mathrm{R}} \lambda_{4}\right) \\ \dot{\lambda}_{1}=-2 \mathrm{Q}_{1}\left(\mathrm{x}_{1}-\mathrm{x}_{1 \mathrm{~d}}\right)+\frac{\tau_{\mathrm{g}}}{\mathrm{I}} \lambda_{2} \cos \left(\mathrm{x}_{1}\right) \\ \dot{\lambda}_{2}=-\lambda_{1}+\frac{\mathrm{B}_{\emptyset}}{\mathrm{I}} \lambda_{2} \\ \dot{\lambda}_{3}=-2 \mathrm{Q}_{3}\left(\mathrm{x}_{3}-\mathrm{x}_{3 \mathrm{~d}}\right)-\frac{2 \mathrm{a}_{1}}{\mathrm{I}} \lambda_{2} \mathrm{x}_{3}-\frac{\mathrm{b}_{1}}{\mathrm{I}} \lambda_{2}+\frac{1}{\mathrm{~T}_{1}^{2}} \lambda_{4} \\ \dot{\lambda}_{4}=-\lambda_{3}+\frac{2 \mathrm{~T}_{1}}{\mathrm{~T}_{1}^{2}} \lambda_{4} \\ \mathrm{u}=\frac{-1}{\mathrm{~T}_{1}^{2} 2 \mathrm{R}} \lambda_{4}\end{array}\right.$

(rv) با شر ايط مرزى $\left\{\begin{array}{l}\mathrm{x}_{1}\left(\mathrm{t}_{0}\right)=\mathrm{x}_{10} \\ \mathrm{x}_{2}\left(\mathrm{t}_{0}\right)=\mathrm{x}_{20} \\ \mathrm{x}_{3}\left(\mathrm{t}_{0}\right)=\mathrm{x}_{30} \\ \mathrm{x}_{4}\left(\mathrm{t}_{0}\right)=\mathrm{x}_{40}\end{array}\right.$ $\left\{\begin{array}{l}2 \mathrm{H}_{1}\left(\mathrm{x}_{1}\left(\mathrm{t}_{\mathrm{f}}\right)-\mathrm{x}_{1 \mathrm{ref}}\right)-\lambda_{1}\left(\mathrm{t}_{\mathrm{f}}\right)=0 \\ \lambda_{2}\left(\mathrm{t}_{\mathrm{f}}\right)=0 \\ 2 \mathrm{H}_{3}\left(\mathrm{x}_{3}\left(\mathrm{t}_{\mathrm{f}}\right)-\mathrm{x}_{3 \mathrm{ref}}\right)-\lambda_{3}\left(\mathrm{t}_{\mathrm{f}}\right)=0 \\ \lambda_{4}\left(\mathrm{t}_{\mathrm{f}}\right)=0\end{array}\right.$ 


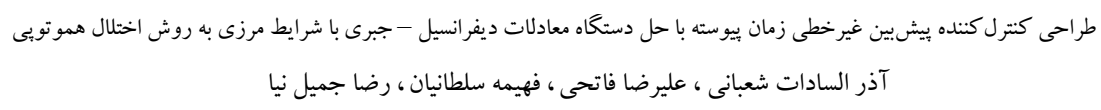

زمان بيوسته است و نشان داده مىشود كه طراحى كنترل كننده ييشبين

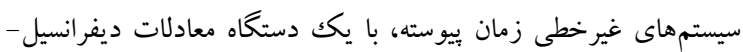

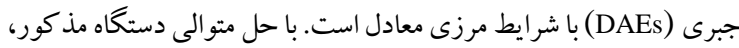

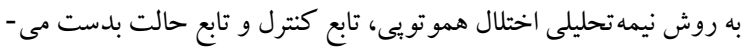

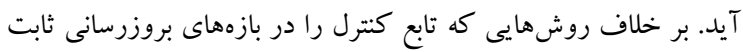

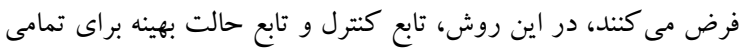

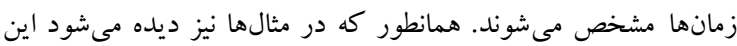

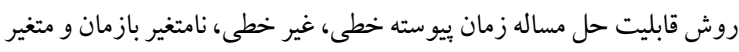

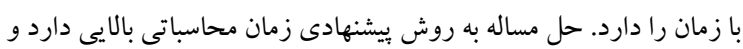

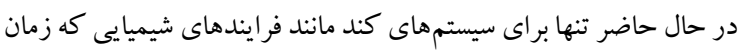

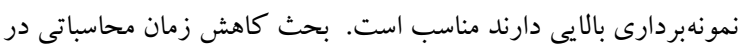

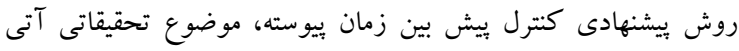

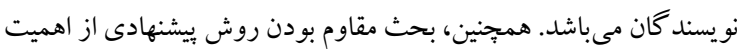

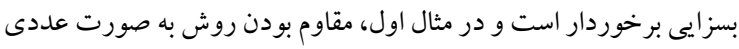

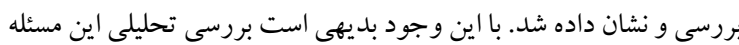
نياز به تحقيقات بيشترى دارد.

\section{مر اجع}

[1] Kirches C., Wirsching L., Bock J.P, 2012, "Efficient direct multiple shooting for nonlinear model predictive control on long horizon", Schloder Journal of Process Control 22, pp. 540-550.

[2] Magni L., Scattolini R., 2004, "Model predictive control of continuouse time nonlinear systems with picewise constant control", IEEE transactions on automatic control, vol. 49, NO. 6, pp. 900 - 906.

[3] Magni L., Scattolini R., 2007, "Tracking of nonsquare nonlinear continuous time systems with piecewise constant model predictive control", Journal of Process Control 17, pp. 631-640.

[4] Wang L., 2001, “continuous time model predictive control design using orthonormal functions", INT.J.control, vol. 74, NO. 16, pp. 15881600 .

[5] Cizniar M., Fikar M., Latifi M.A., 2008, "Design of constrained nonlinear model predictive control based on global optimization", $18^{\text {th }}$ European Symposium on Computer Aided Process Engineering - ESCAPE 18 Bertrand Braunschweig and Xavier Joulia (Editors).

[6] Findeisen R., Raff T., Allgower F., 2007, "Sampled-Data Model Predictive Control for Constrained Continuous Time Systems," Advanced Strategies in Control Systems with Input and Output Contraints, pp.207-235.

[7] Li S.E., Xu SH., Ku D., 2016, "Efficient and accurate computation model predictive control using
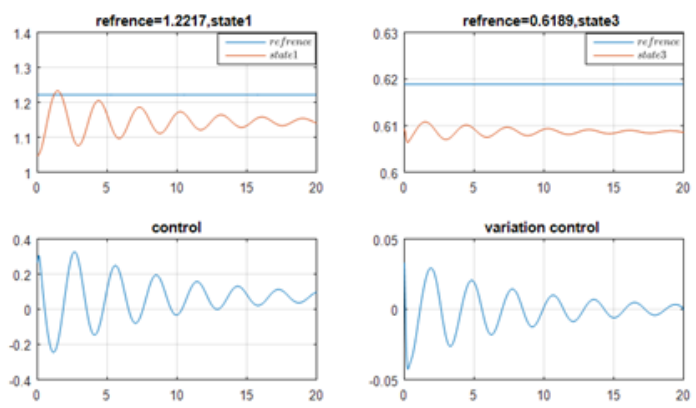

شكل ه) ياسخ كنترل ييشبين زمان بيوسته هليكويتر براى زاويه •Vدرجه
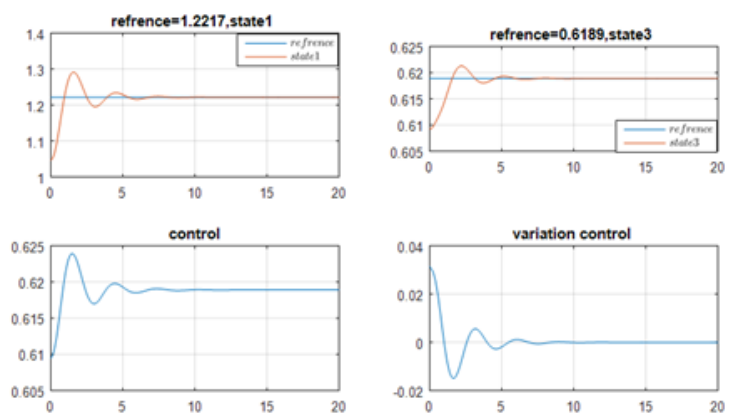

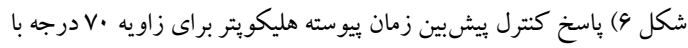
اضافه كردن انتخر ال كير و تنظيم يارامترها
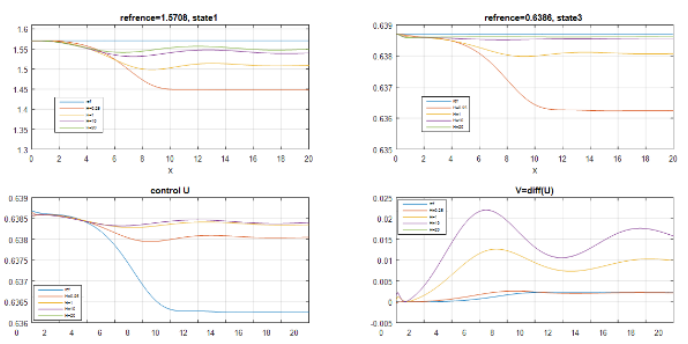

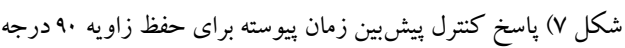

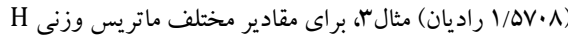

همانطور كه ديده مى شود با طر احى كنترل كننده تو انستهايم وضعيت هلى -

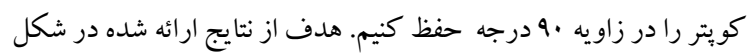

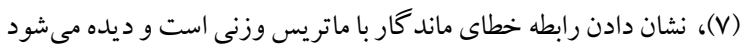
كه با تنظيم ماتريس هاى وزنى مى توان خطاى ماند كار را تنظيم نمود. نتايج براى ماتريسهاى وزنى متفاوت H = 0.25,1,10,20 آورده شده-

\section{0-نتيجه كيرى}

از آنجايى كه در كارهاى انجام شده در زمينه طراحى كنترل كننده

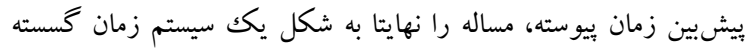

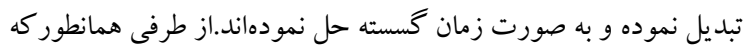

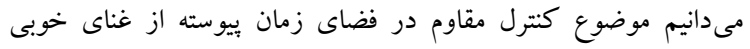

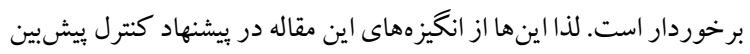




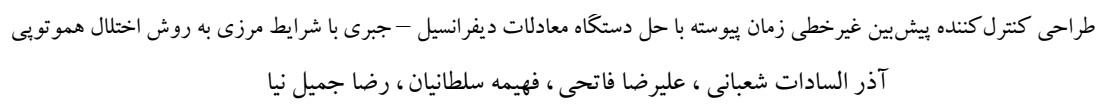

homotopy perturbation method and their engineering applications", International Journal of Computer Mathematics, Vol. 87, No. 9, pp.1950-1974.

[18] Roozi A., Alibeiki E., Hosseini S.S., Ebrahimi M., 2011, "Homotopy perturbation method for special nonlinear partial differential equations", Journal of King Saud University - Science, vol 23, issue 1, pp. 99-103.

[19] Ayati Z., Biazar J., 2015, “On the convergence of Homotopy perturbation Method", Journal of the Egyptian Mathematical Society, vol.23 (2), pp. 424428.

[20] Chen H., Allgower F., 1998, “A Quasi-Infinite Horizon Nonlinear Model Predictive Control Scheme with Guaranteed Stability", Automatica, Vol. 34, No. 10, pp. 1205-1217.

[21] Chen H., Allgower F., 1997, “A quasi infinite horizon nonlinear predictive control scheme for stable", IFAC Proceedings Volumes, Vol. 30, No. 9, pp. 529-534.

[22] Jadbabaie A., Hauser J., 2005, “On the stability of receding horizon control with a general terminal cost", IEEE Transactions on Automati control, Volume 50, issue 5, pp. $674-678$.

[23] Camacho E.F., Bordons C., Model Peredictive Control, Advanced Texbooks in Control and Signal Processing, Springer, Cham, 2004.

[24] Fu H.S, Han B., 2006, A homotopy method for nonlinear inverse problems, Applied Mathematics and Computation, vol.183, pp. 1270-1279.

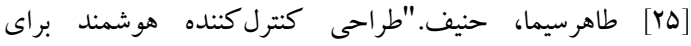

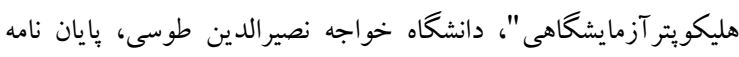

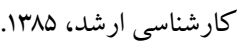

$$
\begin{aligned}
& \text { [Yצ] قربانى، اصغر. "روش اختلال هما. هموتويى هى و كاربرد آن"، } \\
& \text { دانشگاه فردوسى مشهد، يايان نامه كارشناسى ارشد، ومبـا. }
\end{aligned}
$$

pseudospectral discretization", Neurocomputing, 177 , pp. 363-372.

[8] Chen W.H., 2004, "Predictive Control of General Nonlinear Systems Using Approximation", IEE proceedings: control theory and applications, 151 (2), pp. 137-144.

[9] Wang, Y., Boyd, S., (2008), "Fast model predictive control using online optimization", The International Federation of Automatic Control,Vol. 41, pp. 6974-6979.

[10] Pannocchia, G., Rawlings, J. B., Mayne, D. Q., Marquardt, M., (2010)," On computing solutions to the continuous time constrained linear quadratic regulator", IEEE Transactions on Automatic Control, Vol. 55, pp. 2192-2198.

[11] Daehlen, J. S., Otto Eikrem, G., (2014), "Nonlinear model predictive control using trust region derivative free optimization", Journal of Process Control, Vol. 24, pp. 1106-1120.

[12] D.E. Kirk, "Optimal control theory: an introduction", Dover Books on Electical Engineering Series, 2004.

[13] T.J. Böhme, B. Frank, "Direct Methods for Optimal Control", In: Hybrid Systems, Optimal Control and Hybrid Vehicles. Advances in Industrial Control. Springer, Cham, 2017.

[14] Mangasarian O.L., 1996, "sufficient conditions for the optimal control of nonlinear systems", J. SIAM control, Vol. 4, No. 1.

[15] He J. H., 1999 "Homotopy perturbation technique", Computer Methods in Applied Mechanics and Engineering, Vol. 178 (3), pp. 257262.

[16]Aslam Noor M., 2010, "Some iterative methods for solving nonlinear equations using homotopy perturbation method", international journal of computer mathematics, Vol. 87, No. 1, pp. 141-149.

[17] Soltanian F., Dehghan M., Karbassi S.M., 2010, "Solution of the differential algebraic equations via 